\title{
On parameter estimation of stochastic delay differential equations with guaranteed accuracy by noisy observations*
}

\author{
Uwe Küchler \\ Institute of Mathematics \\ Humboldt University Berlin \\ Unter den Linden 6, D-10099 \\ Berlin, Germany
}

\author{
Vjatscheslav A. Vasil'iev \\ Department of Applied Mathematics \\ and Cybernetics \\ Tomsk State University \\ Lenina 36, 634050 Tomsk, Russia
}

\section{Abstract}

Let $(X(t), t \geq-1)$ and $(Y(t), t \geq 0)$ be stochastic processes satisfying

$$
d X(t)=a X(t) d t+b X(t-1) d t+d W(t)
$$

and

$$
d Y(t)=X(t) d t+d V(t)
$$

respectively. Here $(W(t), t \geq 0)$ and $(V(t), t \geq 0)$ are independent standard Wiener processes and $\vartheta=(a, b)^{\prime}$ is assumed to be an unknown parameter from some subset $\Theta$ of $\mathcal{R}^{2}$.

The aim here is to estimate the parameter $\vartheta$ based on continuous observation of $(Y(t), t \geq 0)$.

Sequential estimation plans for $\vartheta$ with preassigned mean square accuracy $\varepsilon$ are constructed using the so-called correlation method. The limit behaviour of the duration of the estimation procedure is studied if $\varepsilon$ tends to zero.

AMS classification: 34K50; 60H10; 62L10; 62L12

Keywords and Phrases: Stochastic delay differential equations; sequential analysis; noisy observations; mean square accuracy

* The research on this paper was supported by RFFI - DFG 02-01-04001 Grant 


\section{Introduction}

Assume $(\Omega, \mathcal{F},(\mathcal{F}(t), t \geq 0), P)$ is a given filtered probability space and the processes $W=(W(t), t \geq 0)$ and $V=(V(t), t \geq 0)$ are real-valued standard Wiener processes on $(\Omega, \mathcal{F},(\mathcal{F}(t), t \geq 0), P)$, adopted to $(\mathcal{F}(t))$ and mutually independent. Furtherer assume that $X_{0}=\left(X_{0}(t), t \in[-1,0]\right)$ and $Y_{0}$ are a real-valued cadlag process and a real-valued random variable, respectively, on $(\Omega, \mathcal{F},(\mathcal{F}(t), t \geq 0), P)$ with

$$
E \int_{-1}^{0} X_{0}^{2}(s) d s<\infty \text { and } E Y_{0}^{2}<\infty .
$$

Assume that $Y_{0}$ and $X_{0}(s)$ are $\mathcal{F}_{0}-$ measurable for every $s$ from $[-1,0]$ and that the quantities $W, V, X_{0}$ and $Y_{0}$ are mutually independent.

Consider a two-dimensional random process $(X, Y)=(X(t), Y(t))$ described by the system of stochastic differential equations

$$
\begin{gathered}
d X(t)=a X(t) d t+b X(t-1) d t+d W(t), t \geq 0, \\
d Y(t)=X(t) d t+d V(t), t \geq 0
\end{gathered}
$$

with the initial conditions $X(t)=X_{0}(t), t \in[-1,0]$ and $Y(0)=Y_{0}$. The process $X$ is supposed to be hidden, i.e. unobservable, and the process $Y$ is observed. Such models are used in applied problems connected with control, filtering and prediction of stochastic processes (see, for example, [1], [7]).

The parameter $\vartheta=(a, b)^{\prime}$ with $a, b \in \mathcal{R}^{1}$ is assumed to be unknown and shall be estimated by using the observation of $Y$.

Equations (1) and (2) together with the initial values $X_{0}(\cdot)$ and $Y_{0}$ respectively have uniquely solutions $X(\cdot)$ and $Y(\cdot)$, for details see [9].

Equation (1) is a very special case of stochastic differential equations with time delay, see [3] and [10] for examples.

To estimate the true parameter $\vartheta$ with a preassigned least square accuracy $\varepsilon$ we shall construct sequential plans $\left(T_{\varepsilon}, \vartheta_{\varepsilon}^{*}\right)$. Moreover, we will derive asymptotic properties of the duration $T_{\varepsilon}$ of these plans for $\varepsilon$ tending to zero.

The method used below is to transform the equations (1) and (2) to a single equation (see (4) below) for the process $(Y(t), t \geq 0)$, which can be treated by modifying a method from [11]. The construction of $\left(T_{\varepsilon}, \vartheta_{\varepsilon}^{*}\right)$ may depend on the asymptotic behaviour of the correlation function of the solution of (1) and their estimators if the observation time is increasing unboundedly. These asymptotic properties vary if $\vartheta$ runs through $\mathcal{R}^{2}$. Our construction does not seem to work for all $\vartheta$ in $\mathcal{R}^{2}$. Therefore we restrict the discussion to two sets $\Theta_{1}$ and $\Theta_{2}$ of parameters, for which we are able to derive the desired properties.

The organization of this paper is as follows. In Section 2 we summarize some known properties of equation (1) needed in the sequel. The two mentioned cases for $\Theta$, namely $\Theta_{1}$ and $\Theta_{2}$, are presented and equations (1), (2) are transformed into a new one for the one-dimensional observed process $(Y(t), t \geq 0$ ) (see (4)). In Section 3 the two sequential plans are constructed and the assertions are formulated. Section 4 contains the proofs. 


\section{Preliminaries}

First we summarize some known facts about equation (1). For details the reader is refer to [2]. Together with the described initial condition equation (1) has a uniquely determined solution $X$ which can be represented as follows for $t \geq 0$ :

$$
X(t)=x_{0}(t) X_{0}(t)+b \int_{-1}^{0} x_{0}(t-s-1) X_{0}(s) d s+\int_{0}^{t} x_{0}(t-s) d W(s), t \geq 0 .
$$

Here $x_{0}=\left(x_{0}(t), t \geq-1\right)$ denotes the so-called fundamental solution of the deterministic equation

$$
x_{0}(t)=1+\int_{0}^{t}\left(\vartheta_{0} x_{0}(s)+\vartheta_{1} x_{0}(s-1)\right) d s, \quad t \geq 0,
$$

corresponding to $(1)$ with $x_{0}(t)=0, \quad t \in[-1,0), \quad x_{0}(0)=1$.

The solution $X$ has the property $E \int_{0}^{T} X^{2}(s) d s<\infty$ for every $T>0$.

The limit behavior of $x_{0}(t)$ and therefore also of $X(t)$ for $t$ tending to infinity is closely connected with the properties of the set $\Lambda=\left\{\lambda \in \mathbb{C} \mid \lambda=a+b e^{-\lambda}\right\}(\mathbb{C}$ denotes the set of complex numbers). The set $\Lambda$ is countable infinite (if $b \neq 0$ ), and for every real $c$ the set $\Lambda_{c}=\Lambda \cap\{\lambda \in \mathbb{C} \mid R e \lambda \geq c\}$ is finite. In particular, $v_{0}:=v_{0}(\vartheta)=\sup \{\operatorname{Re} \lambda \mid \lambda \in \Lambda\}<\infty, \quad \sup \{\emptyset\}=-\infty$. Define $v_{1}(\vartheta)=: \sup \{\operatorname{Re} \lambda \mid \lambda \in$ $\left.\Lambda, \operatorname{Re} \lambda<v_{0}(\vartheta)\right\}$.

The values $v_{0}(\vartheta)$ and $v_{1}(\vartheta)$ determine the asymptotic behaviour of $x_{0}(t)$ as $t \rightarrow$ $\infty$. Indeed, it exist a real $\gamma$ less than $v_{1}$ and a polynomial $\Psi_{1}(\cdot)$ of degree less than or equal one, being specified in the proof of Theorem 3.1 (Section 4 below), such that

$$
x_{0}(t)=\frac{1}{v_{0}-a+1} e^{v_{0} t}+\Psi_{1}(t) e^{v_{1} t}+o\left(e^{\gamma t}\right) \text { as } t \rightarrow \infty .
$$

Now we define a subset $\Theta$ of $\mathcal{R}^{2}$ consisting of two disjoint sets $\Theta_{1}$ and $\Theta_{2}$. First fix a positive real $\bar{\vartheta}$.

Case I. The set $\Theta_{1}$ : Assume $L$ is an arbitrary line in the plane $\mathcal{R}^{2}$ :

$$
L=L(\alpha, \beta, \omega)=\left\{\tilde{\vartheta}=(\tilde{a}, \tilde{b})^{\prime} \mid \alpha \tilde{a}+\beta \tilde{b}=c\right\} .
$$

Let $\tilde{\Theta}$ be the segment $L \cap\{\|\tilde{\vartheta}\| \leq \bar{\vartheta}\}$ (it is no restriction of generality to assume that $\tilde{\Theta}$ is non-void), $\|\cdot\|$ denotes the Euclidean norm.

Now we introduce the set $S$ by

$$
S=\left\{\vartheta=(a, b)^{\prime} \in \tilde{\Theta} \mid v_{0}(\vartheta) \cdot v_{1}(\vartheta)=0 \text { or }\left(a>1, b=-e^{(a-1)}\right)\right\}
$$

and put $\Theta_{1}=\tilde{\Theta} \backslash S$.

Case II. The set $\Theta_{2}$ : Define

$$
\Theta_{2}=\left\{\vartheta \in \mathcal{R}^{2} \mid\|\vartheta\| \leq \bar{\vartheta}, v_{0}(\vartheta)<0 \text { or }\left(v_{0}(\vartheta)>0 \text { and } v_{0}(\vartheta) \notin \Lambda\right)\right\} .
$$


The definition of the two sets $\Theta_{1}$ and $\Theta_{2}$ looks quite complicate. But they are distinguished by the property, that for all of their elements $\vartheta$ the correlation function of $X(\cdot)$ has an asymptotic property which is analogous to (16), (17), (41) and (42) below.

In particular, in Case I the partly observable two-dimensional process $(X(t), Y(t))$ will be reduced to a scalar observable linear process with a scalar function in the dynamic part. The asymptotic properties of this function are given in (16) and (17).

In Case II the information matrix $G_{X}(T)$ given by

$$
G_{X}(T)=\left(\begin{array}{ll}
\int_{0}^{T} X^{2}(t) d t & \int_{0}^{T} X(t) X(t-1) d t \\
T & \int_{0}^{T} X(t) X(t-1) d t \\
\int_{0}^{T} X^{2}(t-1) d t
\end{array}\right)
$$

has the asymptotic property (see [2] and $[5,6]$ for details)

$$
\lim _{T \rightarrow \infty}\left|\varphi^{-1}(T) G_{X}(T)-I_{\infty}(T)\right|=0 \quad P_{\vartheta}-\text { a.s. },
$$

where

$$
\varphi(T)= \begin{cases}T, & \text { if } v_{0}<0, \\ e^{2 v_{0} T}, & \text { if } v_{0}>0, v_{0} \notin \Lambda .\end{cases}
$$

If $v_{0}<0$ then (1) admits a stationary solution and $I_{\infty}(T) \equiv I_{\infty}$ is a constant positive definite $2 \times 2$-matrix (in the sequel we shall call this case the stationary case); if $v_{0}>0$ and $v_{0}(\vartheta) \notin \Lambda$, then $I_{\infty}(T)$ is nondeterministic periodic with the period $\Delta=\pi / \operatorname{Im} \lambda_{0}$, where $\lambda_{0}$ is the unique element of $\Lambda$ with $\operatorname{Re} \lambda_{0}=v_{0}(\vartheta)$ and $\operatorname{Im} \lambda_{0}>0$ (below we refer to this case as the periodic case).

The problem of sequential estimation of $\vartheta$ by observation without noise under the condition (3) was considered in $[5,6]$.

To construct a sequential plan for estimating $\vartheta$ based on the observation of $Y(\cdot)$ we shall apply the idea of a method first used in [11]. To this end we shall reduce equations (1) and (2) to a single one for $Y$.

Using the integrated form of equations (1) and (2) we can get the following equation for the observed process $Y$

$$
\begin{aligned}
d Y(t) & =[a Y(t)+b Y(t-1)] d t+\left[X(0)-a Y(0)-b Y(0)+b \int_{-1}^{0} X_{0}(s) d s\right. \\
& -a V(t)-b V(t-1)+W(t)] d t+d V(t), t \geq 1
\end{aligned}
$$

Thus we have reduced the system (1), (2) to the form

$$
d Y(t)=\vartheta^{\prime} A(t) d t+\xi(t) d t+d V(t)
$$

with

$$
\begin{gathered}
A(t)=(Y(t), Y(t-1))^{\prime} \\
\xi(t)=X(0)-a Y(0)-b Y(0)+b \int_{-1}^{0} X_{0}(s) d s-a V(t)-b V(t-1)+W(t),
\end{gathered}
$$


where the observable process $(A(t), t \geq 0)$ and the noise $\xi=(\xi(t), t \geq 0)$ are some $(\mathcal{F}(t))$-adapted processes. The problem of estimation of $\vartheta$ with guaranteed accuracy in models of the type (4) was considered in [11].

The functions $A(t)$ and $\xi(t)$ are $\mathcal{F}(t)$-measurable for every $t \geq 1$ and a short calculating shows that all conditions of type (3) in [11], consisting of

$$
\begin{gathered}
E \int_{1}^{T}\left(\|A(t)\|_{1}+|\xi(t)|\right) d t<\infty \quad \text { for all } T>1, \\
E[\tilde{\Delta} \xi(t) \mid \mathcal{F}(t-2)]=0, E\left[(\tilde{\Delta} \xi(t))^{2} \mid \mathcal{F}(t-2)\right] \leq \bar{s}^{2}, t \geq 2, \\
\bar{s}^{2}=1+\bar{\vartheta}^{2},\|A\|_{1}=\sum_{i}\left|A_{i}\right|
\end{gathered}
$$

hold in our case. Here $\tilde{\Delta}$ denotes the difference operator defined by $\tilde{\Delta} f(t)=f(t)-$ $f(t-1)$.

Using this operator and the definition of $\xi$ we obtain the following equation:

$$
\begin{aligned}
d \tilde{\Delta} Y(t) & =a \tilde{\Delta} Y(t) d t+b \tilde{\Delta} Y(t-1) d t+\tilde{\Delta} \xi(t) d t \\
& +d V(t)-d V(t-1), t \geq 2
\end{aligned}
$$

with initial condition $\tilde{\Delta} Y(1)=Y(1)-Y_{0}$.

We have reduced the system (1)-(2) to a single differential equation (6) for the observed process $(\tilde{\Delta} Y(t), t \geq 2)$ depending on the unknown parameters $a$ and $b$. The term $\tilde{\Delta} \xi(t)$ also contains $a$ and $b$, but its variance is controllable in certain sense (see formula (5)).

Nevertheless, $a$ and $b$ can not be estimated from (6) by the maximum likelihood or sequential maximum likelihood method given in [2] or [5,6] respectively, because of the appearance of the terms $\tilde{\Delta} \xi(t) d t$ and $d V(t-1)$. Below we shall propose another way following an idea taken from [11].

\section{Results}

\subsection{Sequential estimation procedure I}

Consider the estimation problem of a linear combination $\theta=l^{\prime} \vartheta, \vartheta \in \Theta_{1}$, where $l=\left(l_{1}, l_{2}\right)^{\prime}$ is some known constant vector such that $\sigma=l_{1} \beta-l_{2} \alpha \neq 0$. Here $\alpha$ and $\beta$ are the constants from the definition of the line $L$, defined in Section 2.

We introduce processes $Z_{1}, Z_{2}$ and $\Psi$ by the formulae

$$
\begin{gathered}
d Z_{1}(t)=\sigma^{-1}(\beta d \tilde{\Delta} Y(t)-c \tilde{\Delta} Y(t-1) d t), t \geq 2, \\
d Z_{2}(t)=-\sigma^{-1}(\alpha d \tilde{\Delta} Y(t)-c \tilde{\Delta} Y(t) d t), t \geq 2, \\
\Psi(t)= \begin{cases}\sigma^{-1}(\beta \tilde{\Delta} Y(t)-\alpha \tilde{\Delta} Y(t-1)), & t \geq 2, \\
0, & t<2 .\end{cases}
\end{gathered}
$$


From (6) and from the definition of $\Theta_{1}$ we get for $t \geq 2$ the system of equations

$$
\begin{gathered}
d Z_{1}(t)=a \Psi(t) d t+\beta \sigma^{-1}(\tilde{\Delta} \xi(t) d t+d \tilde{\Delta} V(t)), \\
d Z_{2}(t)=b \Psi(t) d t-\alpha \sigma^{-1}(\tilde{\Delta} \xi(t) d t+d \tilde{\Delta} V(t)), t \geq 2 .
\end{gathered}
$$

Now we obtain an equation for the observable scalar process $Z(t)=l_{1} Z_{1}(t)+$ $l_{2} Z_{2}(t)$ :

$$
d Z(t)=\theta \Psi(t) d t+\tilde{\Delta} \xi(t) d t+d \tilde{\Delta} V(t), t \geq 2
$$

with unknown parameter $\theta$. For $t<2$ we set $Z(t)=0$.

In a similar way as in [11] we can define a sequential plan for the estimation of $\theta$ from $\left\{l^{6} \vartheta \mid \vartheta \in \Theta_{1}\right\}$ with mean square deviation less than a given positive $\varepsilon$. The sequential estimation plans for $\theta$ have been constructed in [11] based on so-called correlation estimators which are generalized least squares estimators. Here we use an analogous definition as follows:

$$
\begin{gathered}
\theta^{*}(T)=G^{-1}(T, u) \Phi(T, u), \\
G(T, u)=\int_{0}^{T} \Psi(t-u) \Psi(t) d t, \quad \Phi(T, u)=\int_{0}^{T} \Psi(t-u) d Z(t), \quad T>2, \quad u \geq 2 .
\end{gathered}
$$

Under the condition $u \geq 2$ the function $\Psi(t-u)$ in equation (7) is uncorrelated with respect to the noise $\tilde{\tilde{\Delta}} \xi(t)$ as well as to $\tilde{\Delta} V(t)$.

From (7) and (8) we find the deviation of the estimator $\theta^{*}(T)$ :

$$
\theta^{*}(T)-\theta=G^{-1}(T, u) \zeta(T, u),
$$

where

$$
\zeta(T, u)=\zeta(T, u, 1)+\zeta(T, u, 2)+\zeta(T, u, 3)
$$

with

$$
\zeta(T, u, 1)=\int_{0}^{T} \Psi(t-u) \tilde{\Delta} \xi(t) d t, \quad \zeta(T, u, 2)=\int_{0}^{T} \Psi(t-u) d V(t)
$$

and

$$
\zeta(T, u, 3)=-\int_{0}^{T} \Psi(t-u) d V(t-1) .
$$

As we will see from the proof of Theorem 3.1 (Section 4 below), there exist increasing functions $\varphi(T)$ corresponding to the various regions for the parameter $\vartheta$ from $\Theta_{1}$ and $\Theta_{2}$ such that for every $u \geq 2$ the function $g(T, u)=\varphi^{-1}(T) G(T, u)$ has one of the following properties: either 
a) the limit $g(u)=\lim _{T \rightarrow \infty} g(T, u)$ exists $P$-a.s. and is deterministic with meas $\{u \in$ $(2,3]: g(u)=0\}=0($ meas $\{B\}$ is the Lebesgue measure of the set $B)$ and $g(0)>0$; or

b) the limit $g(u)=\lim _{T \rightarrow \infty} g(T, u)$ exists $P$ - a.s. and is non-deterministic, it holds $P\{g(u)=0\}=0, u \geq 0$;

or

c) there exists a random periodic function $\tilde{g}(T, u), T>0$, periodic with respect to $T$ and with period $\Delta>1$, such that

$$
P\left\{\lim _{T \rightarrow \infty}|g(T, u)-\tilde{g}(T, u)|=0\right\}=1, u \geq 0
$$

holds (see the formulae (16) and (17) below).

It will be clear from the proofs in Section 4 below that in the periodic case c) the function $\tilde{g}(T, u)$ has for every $u \geq 0$ two roots as a maximum on every interval of the unknown period length $\Delta$. Then the function $\varphi(T) G^{-1}(T, u)$ and consequently the deviation $\theta^{*}(T)-\theta$ may be unbounded.

Remark 1 Properties a) and c) do not exclude that the limit functions $g(u)$ and $\tilde{g}(T, u)$ may be equal to zero for some $u$ and $(T, u)$ respectively. A similar picture arises in Case II (see proof of Theorem 3.2 below). Due to this fact the estimation procedure, used in [11] can not be applied in the cases considered above.

To exclude this effect we introduce a discretization of the time of observations. Note that in the case of observations without noise we also need a similar discretization (by using $\Delta$ ) for the investigation of asymptotic properties of maximum likelihood estimators [2]. The procedure which we construct here is non-asymptotic and we can not use the unknown value $\Delta$ in the construction of estimators.

For some $h \in(0,1 / 3]$ put

$$
r_{n}=\arg \max _{k=1,3}|G(n h-k h, 2+3 h)| .
$$

Such a choice of the value of $h$ implies that for every $n \geq 1$ and $T>0$ there are one or more values $n h-k h, k=\overline{1,3}$, with $\tilde{g}(n h-k h, T) \neq 0$. In such a way (see the proof of Theorem 3.1) the sequence $\left\{g\left(n h-r_{n} h, 2+3 h\right), n \geq 1\right\}$ is non-degenerate in the case c) for any $h \in(0,1 / 3]$ asymptotically as $n \rightarrow \infty$.

To construct the estimators with preassigned accuracy we first change first the value $n h$ in the argument of $G$ (see the definition of $r_{n}$ just given) to stopping times. As we will see later (inequalities (11)) this substitution gives us the possibility to control the second moments of the noise $\zeta$.

Let $\left(c_{n}, n \geq 1\right)$ be some unboundedly increasing sequence of positive numbers. We shall define the stopping times $\left(\tau_{\varepsilon}(n), n \geq 1\right)$ from the discrete sequence $\{k h, k \geq$ $1\}$ with an arbitrary but fixed step size $h$ by formula

$$
\tau_{\varepsilon}(n)=h \inf \left\{k \geq 1: \int_{0}^{k h} \Psi^{2}(t-2-3 h) d t \geq \varepsilon^{-1} c_{n}\right\}, n \geq 1 .
$$

Using formulae (16) and (17) below it is easy to see that $P\left(\tau_{\varepsilon}(n)<\infty\right)=1$ for any $\varepsilon>0$ and every $n \geq 1$. 
For $k=\overline{1,3}, n \geq 1$ we put

$$
\begin{gathered}
G_{\varepsilon}(n, k)=G\left(\tau_{\varepsilon}(n)-k h, 2+3 h\right), \quad \Phi_{\varepsilon}(n, k)=\Phi_{\varepsilon}\left(\tau_{\varepsilon}(n)-k h, 2+3 h\right), \\
\zeta_{\varepsilon}(n, k)=\zeta\left(\tau_{\varepsilon}(n)-k h, 2+3 h\right) ; \\
k_{n}=\arg \max _{k=1,3}\left\{\left|G_{\varepsilon}(n, k)\right|\right\}, n \geq 1 .
\end{gathered}
$$

Now we introduce the sequence of estimators

$$
\theta_{\varepsilon}(n)=G_{\varepsilon}^{-1}(n) \Phi_{\varepsilon}(n)
$$

with

$$
G_{\varepsilon}(n)=G_{\varepsilon}\left(n, k_{n}\right), \quad \Phi_{\varepsilon}(n)=\Phi\left(n, k_{n}\right), n \geq 1 .
$$

They have the deviation

$$
\theta_{\varepsilon}(n)-\theta=G_{\varepsilon}^{-1}(n) \zeta_{\varepsilon}(n), \quad \zeta_{\varepsilon}(n)=\zeta_{\varepsilon}\left(n, k_{n}\right), n \geq 1 .
$$

Fix an $h_{0}$ from $(0,1 / 3)$ and choose an arbitrary random variable $h$ being $\mathcal{F}(0)$ measurable and having a continuous distribution concentrated on the interval $\left[h_{0}, 1 / 3\right]$. We need such randomization of the discretization step $h$ in the case a) for the almost surely non-degeneration of the limit $g(2+3 h)=\lim _{n \rightarrow \infty} \varphi^{-1}\left(\tau_{\varepsilon}(n)-k_{n} h\right) G_{\varepsilon}\left(n, k_{n}\right)$.

We will show that the second moments of the noise $\zeta$ calculated at times $\tau_{\varepsilon}(n)-$ $k_{n} h, n \geq 1$ have known upper bounds. Note that the processes $(\zeta(T, 2+3 h, i), \mathcal{F}(T))$, $i=\overline{1,3}$ are square integrable martingales and the times $\tau_{\varepsilon}(n)-k h, n \geq 1, k=\overline{1,3}$, are Markovian with respect to the system $(\mathcal{F}(T-2))$. From the theory of martingales (see e.g. [8]) and from the definition of $\tau_{\varepsilon}(n)$ we obtain for all $\vartheta \in \mathcal{R}^{2}, k=\overline{1,3}$ and $n \geq 1$ the inequalities

$$
\begin{gathered}
E_{\vartheta} \zeta^{2}\left(\tau_{\varepsilon}(n)-k h, 2+3 h, 1\right) \leq \bar{s}^{2} E_{\vartheta} \int_{0}^{\tau_{\varepsilon}(n)-k h} \Psi^{2}(t-2-3 h) d t<\bar{s}^{2} \varepsilon^{-1} c_{n}, \\
E_{\vartheta} \zeta^{2}\left(\tau_{\varepsilon}(n)-k h, 2+3 h, i\right) \leq \varepsilon^{-1} c_{n}, i=2,3 .
\end{gathered}
$$

Thus for all $\varepsilon>0$ and $n \geq 1$ the sequence $\left(\zeta_{\varepsilon}(n), n \geq 1\right)$ satisfies the inequalities

$$
\begin{aligned}
E_{\vartheta} \zeta_{\varepsilon}^{2}(n) & \leq \sum_{k=1}^{3} E_{\vartheta} \zeta^{2}\left(\tau_{\varepsilon}(n)-k h, 2+3 h\right) \\
& \leq 3 \sum_{k=1}^{3} \sum_{i=1}^{3} E_{\vartheta} \zeta^{2}\left(\tau_{\varepsilon}(n)-k h, 2+3 h, i\right) \leq 9\left(2+\bar{s}^{2}\right) \varepsilon^{-1} c_{n} .
\end{aligned}
$$

The asymptotic properties of the sequence $\left(G_{\varepsilon}(n), n \geq 1\right)$ and the inequalities (11) imply that the estimation of the parameter $\theta$ should be performed at the times $\tau_{\varepsilon}(n)-k_{n} h, n \geq 1$. Note that the estimators $\theta_{\varepsilon}(n)$ are strongly consistent (see 
Theorem 3.1).

We want obtain estimators with fixed mean square deviation. Therefore, taking into account the representation for the deviation of estimators $\theta_{\varepsilon}(n)$, one has to control the behaviour of the sequence of random variables $G_{\varepsilon}(n), n \geq 1$. This can be achieved by observations up to the time $\tau_{\varepsilon}(n)-k_{n} h$ with a specially chosen number $n$.

Let $\left(\kappa_{n}, n \geq 1\right)$ be some unboundedly increasing sequence of positive numbers. Introduce the stopping time

$$
\nu_{\varepsilon}=\inf \left\{n \geq 1:\left|G_{\varepsilon}(n)\right| \geq \rho^{1 / 2} \varepsilon^{-1} \kappa_{n}\right\},
$$

where

$$
\rho=9\left(2+\bar{s}^{2}\right) \sum_{n \geq 1} c_{n} / \kappa_{n}^{2}
$$

We define the sequential plan $\left(T(\varepsilon), \theta_{\varepsilon}^{*}\right)$ for the estimation of $\theta$ as

$$
T(\varepsilon)=\tau_{\varepsilon}\left(\nu_{\varepsilon}\right), \theta_{\varepsilon}^{*}=\theta_{\varepsilon}\left(\nu_{\varepsilon}\right)=G_{\varepsilon}^{-1}\left(\nu_{\varepsilon}\right) \Phi_{\varepsilon}\left(\nu_{\varepsilon}\right) .
$$

It should be pointed out that the estimator (12) coincides with the sequential estimator which is obtained from general least squares criteria [11].

The following theorem presents the conditions under which $T(\varepsilon)$ and $\theta_{\varepsilon}^{*}$ are welldefined and have the desired property of preassigned mean square accuracy.

First we divide the parameter set $\Theta_{1}$ into nine subsets, according to the definitions of Section I.

Define the functions $u(a), a<1$, and $w(a), a \in \mathcal{R}^{1}$, as in [2]: consider a parametric curve $(a(\xi), b(\xi)), \xi>0, \xi \neq \pi, 2 \pi, \ldots$, in $\mathcal{R}^{2}$ by

$$
a(\xi)=\xi \cot \xi, b(\xi)=-\xi / \sin \xi,
$$

then functions $b=u(a)$ and $b=w(a)$ are defined to be the branches of this curve corresponding to $\xi \in(0, \pi)$ and $\xi \in(\pi, 2 \pi)$ respectively. Put also $v(a)=-e^{a-1}, a \in$ $\mathcal{R}^{1}$, and introduce the indices

$$
\begin{gathered}
i= \begin{cases}0, & \text { if } \alpha \neq \beta e^{v_{0}} \\
1, & \text { if } \alpha=\beta e^{v_{0}},\end{cases} \\
j= \begin{cases}1, & \text { if } a<1, u(a)<b<-a, \\
2, & \text { if }-a<b<w(a), \\
3, & \text { if } a>1, v(a)<b<-a, \\
4, & \text { if } a>1, b=v(a), \\
5, & \text { if } b>w(a), \\
6, & \text { if } a<1, b<u(a) \text { or } a \geq 1, b<v(a), \\
7, & \text { if } a<1, b=-a, a \neq 0, \\
8, & \text { if } a>1, b=-a, \\
9, & \text { if } b=w(a) .\end{cases}
\end{gathered}
$$


Note that the sets corresponding to different values of $j$ are disjoint and the union of all the cases corresponding to $j=\overline{1,9}$ is the whole plane $\mathcal{R}^{2}$ exept for some one-dimensional smooth curve. We know that $v_{0}<0$ if $j=1 ; v_{0}=0$ if $j=7$ and $v_{0}>0$ in all other cases. Moreover we have $v_{1}<0$ if $j=1,2,7 ; v_{1}=0$ if $j=8,9$ and $v_{1}>0$ if $j=3,5[2]$.

Introduce the sets

$$
\begin{gathered}
I_{1}=\{(0,1),(1,1),(1,2),(1,7)\}, \\
I_{2}=\{(0,2),(0,3),(0,5),(0,8),(0,9),(1,4)\}, \\
I_{3}=\{(1,3)\}, \quad I_{4}=\{(0,4)\}, \quad I_{5}=\{(0,6),(1,5),(1,6)\}, \\
I_{6}=I_{2} \cup I_{5} \backslash\{(1,5)\}, \quad I_{7}=I_{3} \cup\{(1,5)\} .
\end{gathered}
$$

Theorem 3.1 Assume that the sequences $\left(c_{n}\right)$ and $\left(\kappa_{n}\right)$ defined above satisfy the conditions

$$
\sum_{n \geq 1} \frac{c_{n}}{\kappa_{n}^{2}}<\infty
$$

and

$$
\lim _{n \rightarrow \infty} \kappa_{n} / c_{n}=0
$$

Then we obtain the following result:

I. For any $\varepsilon>0$ and every $\theta \in \Theta_{1}$ the sequential plan $\left(T(\varepsilon), \theta_{\varepsilon}^{*}\right)$ defined by (12) is closed (i.e. $T(\varepsilon)<\infty P-$ a.s.) and has the following properties:

$$
1^{\circ} . \sup _{\Theta_{1}} E_{\vartheta}\left(\theta_{\varepsilon}^{*}-\theta\right)^{2} \leq \varepsilon \quad \text { for every } \varepsilon>0,
$$

$2^{\circ}$. for every $\theta \in \Theta_{1}$ the following relations hold:

- if $(i, j) \in I_{1}$ then

$$
0<\varliminf_{\varepsilon \rightarrow 0} \varepsilon \cdot T(\varepsilon) \leq \varlimsup_{\varepsilon \rightarrow 0} \varepsilon \cdot T(\varepsilon)<\infty P-\text { a.s. }
$$

- if $(i, j) \in I_{2} \cup I_{3} \cup I_{5}$ then

$$
0<\lim _{\varepsilon \rightarrow 0}\left[T(\varepsilon)-\frac{1}{2 v_{i}} \ln \varepsilon^{-1}\right] \leq \varlimsup_{\varepsilon \rightarrow 0}\left[T(\varepsilon)-\frac{1}{2 v_{i}} \ln \varepsilon^{-1}\right]<\infty P-\text { a.s. }
$$

- if $(i, j) \in I_{4}$ then

$$
0<\lim _{\varepsilon \rightarrow 0} \varepsilon T^{2}(\varepsilon) e^{2 v_{0} T(\varepsilon)} \leq \varlimsup_{\varepsilon \rightarrow 0} \varepsilon T^{2}(\varepsilon) e^{2 v_{0} T(\varepsilon)}<\infty \quad P-\text { a.s. }
$$

II. For any $\varepsilon>0$ and every $\theta \in \Theta_{1}$ the estimator $\theta_{\varepsilon}(n)$ is strongly consistent:

$$
\lim _{n \rightarrow \infty} \theta_{\varepsilon}(n)=\theta \quad P-\text { a.s. }
$$

The proofs of this and the next theorem are given in Section 4 .

Remark 2 Consider the special case of the system (1),(2), when the parameter $b$ equals zero, which means that $X(\cdot)$ is an Ornstein-Uhlenbeck process. Then the assertions of Theorem 3.1 are true if in equation (1) we have $a \neq 0$. Note, that in [11] only the case $(a<0)$ has been considered. 


\subsection{Sequential estimation procedure II}

Consider the problem of estimating $\vartheta \in \Theta_{2}$. Based on equation (6) we define the estimation procedure analogously to the one given in Section 3.1. Assume $\tilde{h}_{0}$ is a real number in $(0,1 / 5)$ and $\tilde{h}$ is a random variable with values in $\left[\tilde{h}_{0}, 1 / 5\right]$ only, $\mathcal{F}(0)$-measurable and having a known continuous distribution function.

We introduce several quantities:

- the functions

$$
\tilde{\Psi}_{s}(t)=\left\{\begin{array}{l}
(\tilde{\Delta} Y(t), \tilde{\Delta} Y(t-s))^{\prime} \quad \text { for } \quad t \geq 1+s \\
(0,0)^{\prime} \text { for } t<1+s
\end{array}\right.
$$

- the sequence of stopping times

$$
\tilde{\tau}_{\varepsilon}(n)=\tilde{h} \inf \left\{k \geq 1: \int_{0}^{k \tilde{h}}\left\|\tilde{\Psi}_{\tilde{h}}(t-2-5 \tilde{h})\right\|^{2} d t \geq \varepsilon^{-1} c_{n}\right\} \quad \text { for } \quad n \geq 1 ;
$$

- the matrices

$$
\begin{gathered}
G^{*}(T, s)=\int_{0}^{T} \tilde{\Psi}_{s}(t-2-5 s) \tilde{\Psi}_{1}^{\prime}(t) d t, \\
\Phi^{*}(T, s)=\int_{0}^{T} \tilde{\Psi}_{s}(t-2-5 s) d \tilde{\Delta} Y(t), \\
\tilde{G}_{\varepsilon}(n, k)=G^{*}\left(\tilde{\tau}_{\varepsilon}(n)-k \tilde{h}, \tilde{h}\right), \quad \tilde{\Phi}_{\varepsilon}(n, k)=\Phi^{*}\left(\tilde{\tau}_{\varepsilon}(n)-k \tilde{h}, \tilde{h}\right) ;
\end{gathered}
$$

- the times

$$
\tilde{k}_{n}=\arg \min _{k=1,5}\left\|\tilde{G}_{\varepsilon}^{-1}(n, k)\right\|, n \geq 1 ;
$$

- the estimators

$$
\begin{aligned}
& \tilde{\vartheta}_{\varepsilon}(n)=\tilde{G}_{\varepsilon}^{-1}(n) \tilde{\Phi}_{\varepsilon}(n), n \geq 1, \quad \text { where } \\
& \tilde{G}_{\varepsilon}(n)=\tilde{G}_{\varepsilon}\left(n, \tilde{k}_{n}\right), \tilde{\Phi}_{\varepsilon}(n)=\tilde{\Phi}_{\varepsilon}\left(n, \tilde{k}_{n}\right) ;
\end{aligned}
$$

- the stopping time

$$
\begin{gathered}
\tilde{\nu}_{\varepsilon}=\inf \left\{n \geq 1:\left\|\tilde{G}_{\varepsilon}^{-1}(n)\right\| \leq \varepsilon\left(\tilde{\rho}^{1 / 2} \kappa_{n}\right)^{-1}\right\}, \quad \text { where } \\
\tilde{\rho}=15\left(2+\bar{s}^{2}\right) \sum_{n \geq 1} c_{n} / \kappa_{n}^{2} .
\end{gathered}
$$

Define the sequential estimation plan of $\vartheta$ by

$$
\tilde{T}(\varepsilon)=\tilde{\tau}_{\varepsilon}\left(\tilde{\nu}_{\varepsilon}\right), \quad \tilde{\vartheta}(\varepsilon)=\tilde{\vartheta}_{\varepsilon}\left(\tilde{\nu}_{\varepsilon}\right)=\tilde{G}_{\varepsilon}^{-1}\left(\tilde{\nu}_{\varepsilon}\right) \tilde{\Phi}_{\varepsilon}\left(\tilde{\nu}_{\varepsilon}\right) .
$$


We can see that the construction of the sequential estimator $\tilde{\vartheta}(\varepsilon)$ bases on the family of estimators $\vartheta^{*}(T, s)=\left(G^{*}(T, s)\right)^{-1} \Phi^{*}(T, s), s \geq 0$. We have taken the discretization step $\tilde{h}$ as above, because from (49) below it follows that the functions

$$
\tilde{f}(T, s)=\frac{1}{e^{2 v_{0} T}} G^{*}(T, s)
$$

for every $s \geq 0$ have some periodic matrix functions as a limit almost surely. These limiting matrix functions are finite and may be degenerate only for four values of their argument $T$ on every interval of periodicity of length $\Delta>1$ (see proof of Theorem 3.2 below).

We state the results concerning the estimation of the parameter $\vartheta \in \Theta_{2}$ in the following theorem.

Theorem 3.2 Assume that the conditions (13) and (14) on the sequences $\left(c_{n}\right)$ and $\left(\kappa_{n}\right)$ hold and let the parameter $\vartheta=(a, b)^{\prime}$ in (1) is such that $\vartheta \in \Theta_{2}$. Then we obtain:

I. For any $\varepsilon>0$ and every $\vartheta \in \Theta_{2}$ the sequential plan $(\tilde{T}(\varepsilon), \tilde{\vartheta}(\varepsilon))$ defined by (15) is closed and possesses the following properties:

$$
1^{o} . \quad \sup _{\Theta_{2}} E_{\vartheta}\|\tilde{\vartheta}(\varepsilon)-\vartheta\|^{2} \leq \varepsilon \quad \text { for every } \varepsilon>0,
$$

$2^{\circ}$. for every $\theta \in \Theta_{2}$ one of the inequalities below is valid:

- in the stationary case $\left(v_{0}<0\right)$

$$
0<\varliminf_{\varepsilon \rightarrow 0} \varepsilon \cdot \tilde{T}(\varepsilon) \leq \varlimsup_{\varepsilon \rightarrow 0} \varepsilon \cdot \tilde{T}(\varepsilon)<\infty P-\text { a.s. }
$$

- in the periodic case $\left(v_{0}>0, v_{0} \notin \Lambda\right)$

$$
0<\lim _{\varepsilon \rightarrow 0}\left[\tilde{T}(\varepsilon)-\frac{1}{2 v_{0}} \ln \varepsilon^{-1}\right] \leq \varlimsup_{\varepsilon \rightarrow 0}\left[\tilde{T}(\varepsilon)-\frac{1}{2 v_{0}} \ln \varepsilon^{-1}\right]<\infty P-\text { a.s. }
$$

II. For any $\varepsilon>0$ and every $\vartheta \in \Theta_{2}$ the estimator $\vartheta_{\varepsilon}(n)$ is strongly consistent:

$$
\lim _{n \rightarrow \infty} \tilde{\vartheta}_{\varepsilon}(n)=\vartheta \quad P-\text { a.s. }
$$

Remark 3 Property I in Theorems 3.1 and 3.2 yields the rates of convergence of the considered sequential plans. These depend on the region to which the parameter $\vartheta$ belongs to. They have the same rate of convergence as the maximum likelihood estimator of $\vartheta$, see [2], constructed directly from the observations of the process $X(\cdot)$.

\section{Proofs}

\subsection{Proof of Theorem 3.1}

At first we prove the finiteness of the stopping times $T(\varepsilon)$.

To this aim we put 


$$
\varphi_{i j}(T)= \begin{cases}T, & (i, j) \in I_{1}, \\ e^{2 v_{i} T}, & (i, j) \in I_{2} \cup I_{3} \cup I_{5}, \\ T^{2} e^{2 v_{0} T}, & (i, j) \in I_{4} .\end{cases}
$$

and prove the following auxiliary results: Fix $u=0$ or $u \in[2, \infty)$. Then - for $(i, j) \in I_{1} \cup I_{2} \cup I_{3} \cup I_{4}$ it holds

$$
\lim _{T \rightarrow \infty} \frac{1}{\varphi_{i j}(T)} \int_{0}^{T} \Psi(t-u) \Psi(t) d t=f_{i j u} \quad P-\text { a.s. },
$$

where $f_{i j u}$ are some constants or random variables;

- for $(i, j) \in I_{5}$ we have

$$
\lim _{T \rightarrow \infty}\left|\frac{1}{\varphi_{i j}(T)} \int_{0}^{T} \Psi(t-u) \Psi(t) d t-f_{i j u}(T)\right|=0 \quad P \text { - a.s. }
$$

where $f_{i j u}(T)$ are periodic random functions of $T$ with the period $\Delta=2 \pi / \xi_{0}, \xi_{0} \in$ $(0, \pi)$ if $(i, j)=\{(0,6),(1,6)\}$ and $\Delta=2 \pi / \xi_{1}, \xi_{1} \in(\pi, 2 \pi)$ if $(i, j)=(1,5)$.

Proof of (16) and (17). Now we establish the equalities (16) in the cases $I_{1}$ for $u=0, u \geq 2$ and the other equalities in (16) and (17) for $u \geq 0$. According to [2] for $\vartheta \in \Theta_{1}$ the solution $X(t)$ of (1) has the representation

$$
\begin{aligned}
X(t) & =x_{0}(t) X_{0}(0)+b \int_{-1}^{0} x_{0}(t-s-1) X_{0}(s) d s \\
& +\int_{0}^{t} x_{0}(t-s) d W(s), t \geq 0,
\end{aligned}
$$

where $x_{0}(\cdot)$ is the so called fundamental solution of (1). It has the properties $x_{0}(t)=$ $0, t \in[-1,0), x_{0}(0)=1$ and satisfies for $t \rightarrow \infty$

$$
x_{0}(t)= \begin{cases}o\left(e^{\gamma t}\right), & \gamma<0, \quad j=1, \\ \frac{1}{v_{0}-a+1} e^{v_{0} t}+o\left(e^{\gamma t}\right), & \gamma<0, j=2, \\ \frac{1}{v_{0}-a+1} e^{v_{0} t}+\frac{1}{a-v_{1}-1} e^{v_{1} t}+o\left(e^{\gamma_{1} t}\right), & \gamma_{1}<v_{1}, j=3, \\ \left(2 t+\frac{2}{3}\right) e^{v_{0} t}+o\left(e^{\gamma_{0} t}\right), & \gamma_{0}<v_{0}, j=4, \\ \frac{1}{v_{0}-a+1} e^{v_{0} t}+\phi_{1}(t) e^{v_{1} t}+o\left(e^{\gamma_{1} t}\right), & \gamma_{1}<v_{1}, j=5, \\ \phi_{0}(t) e^{v_{0} t}+o\left(e^{\gamma_{0} t}\right), & \gamma_{0}<v_{0}, j=6, \\ \frac{1}{1-a}+o\left(e^{\gamma t}\right), & \gamma<0, \quad j=7, \\ \frac{1}{v_{0}-a+1} e^{v_{0} t}-\frac{1}{a-1}+o\left(e^{\gamma t}\right), & \gamma<0, \quad j=8, \\ \frac{1}{v_{0}-a+1} e^{v_{0} t}+\phi_{1}(t)+o\left(e^{\gamma t}\right), & \gamma<0, \quad j=9,\end{cases}
$$

for all $\gamma, \gamma_{0}, \gamma_{1}$ satisfying the mentioned inequalities respectively and may be different in different lines,

$$
\begin{gathered}
\phi_{i}(t)=A_{i} \cos \xi_{i} t+B_{i} \sin \xi_{i} t \text { with } \\
A_{i}=\frac{2\left(v_{i}-a+1\right)}{\left(v_{i}-a+1\right)^{2}+\xi_{i}^{2}}, B_{i}=\frac{2 \xi_{i}}{\left(v_{i}-a+1\right)^{2}+\xi_{i}^{2}}, \quad i=0,1 .
\end{gathered}
$$


By the definition of $\Psi$ we have

$$
\begin{gathered}
\Psi(t)=\tilde{\Psi}(t)+\tilde{V}(t), t \geq-1, \\
\tilde{\Psi}(t)= \begin{cases}\sigma^{-1}(\beta \tilde{X}(t)-\alpha \tilde{X}(t-1)), & t \geq 2, \\
0, & t \in[-1,2],\end{cases} \\
\tilde{X}(t)=\int_{t-1}^{t} X(s) d s, \\
\tilde{V}(t)= \begin{cases}\sigma^{-1}(\beta \tilde{\Delta} V(t)-\alpha \tilde{\Delta} V(t-1)), & t \geq 2, \\
0, & t \in[-1,2] .\end{cases}
\end{gathered}
$$

It is easy to show that the process $(\tilde{X}(\cdot))$ has the following representation:

$$
\tilde{X}(t)=\sigma^{-1}\left(\tilde{x}_{0}(t) X_{0}(0)+b \int_{-1}^{0} \tilde{x}_{0}(t-s-1) X_{0}(s) d s+\int_{0}^{t} \tilde{x}_{0}(t-s) d W(s)\right)
$$

for $t \geq 1, \tilde{X}(t)=\int_{t-1}^{0} X_{0}(s) d s+\int_{0}^{t} X(s) d s$ for $t \in[0,1)$ and $\tilde{X}(t)=0$ for $t \in$ $[-1,0)$. Based on (18) and the subsequent properties of $x_{0}(t)$ the function $\tilde{x}_{0}(t)=$ $\int_{t-1}^{t} x_{0}(s) d s$ can easily be shown to fulfill $\tilde{x}_{0}(t)=0, t \in[-1,0]$ and as $t \rightarrow \infty$

$$
\tilde{x}_{0}(t)= \begin{cases}o\left(e^{\gamma t}\right), & \gamma<0, \quad j=1, \\ \frac{1-e^{-v_{0}}}{v_{0}\left(v_{0}-a+1\right)} e^{v_{0} t}+o\left(e^{\gamma t}\right), & \gamma<0, \quad j=2, \\ \frac{1-e^{-v_{0}}}{v_{0}\left(v_{0}-a+1\right)} e^{v_{0} t}+\frac{1-e^{-v_{1}}}{v_{1}\left(a-v_{1}-1\right)} e^{v_{1} t}+o\left(e^{\gamma_{1} t}\right), & \gamma_{1}<v_{1}, j=3, \\ \frac{2}{v_{0}}\left[\left(1-e^{-v_{0}}\right) t+e^{-v_{0}}-\frac{1-e^{-v_{0}}}{v_{0}}\right] e^{v_{0} t}+o\left(e^{\gamma_{0} t}\right), & \gamma_{0}<v_{0}, j=4, \\ \frac{1-e_{0}}{v_{0}\left(v_{0}-a+1\right)} e^{v_{0} t}+\tilde{\phi}_{1}(t) e^{v_{1} t}+o\left(e^{\gamma_{1} t}\right), & \gamma_{1}<v_{1}, j=5, \\ \tilde{\phi}_{0}(t) e^{v_{0} t}+o\left(e^{\gamma_{0} t}\right), & \gamma_{0}<v_{0}, j=6, \\ \frac{1}{1-a}+o\left(e^{\gamma t}\right), & \gamma<0, \quad j=7, \\ \frac{1-e^{-v_{0}}}{v_{0}\left(v_{0}-a+1\right)} e^{v_{0} t}-\frac{1}{a-1}+o\left(e^{\gamma t}\right), & \gamma<0, \quad j=8, \\ \frac{1-e^{-v_{0}}}{v_{0}\left(v_{0}-a+1\right)} e^{v_{0} t}+\tilde{\phi}_{1}(t)+o\left(e^{\gamma t}\right), & \gamma<0, \quad j=9,\end{cases}
$$

where

$$
\begin{aligned}
& \tilde{\phi}_{i}(t)=\tilde{A}_{i} \cos \xi_{i} t+\tilde{B}_{i} \sin \xi_{i} t \\
& \tilde{A}_{i}= \frac{1}{v_{i}^{2}+\xi_{i}^{2}}\left[\xi_{i} e^{-v_{i}} \sin \xi_{i}-v_{i} e^{-v_{i}} \cos \xi_{i}+v_{i}\right] A_{i} \\
&+ \frac{1}{v_{i}^{2}-\xi_{i}^{2}}\left[v_{i} e^{-v_{i}} \sin \xi_{i}+v_{i} e^{-v_{i}} \cos \xi_{i}-\xi_{i}\right] B_{i}, \\
& \tilde{B}_{i}= \frac{1}{v_{i}^{2}+\xi_{i}^{2}}\left[\xi_{i}-v_{i} e^{-v_{i}} \sin \xi_{i}-\xi_{i} e^{-v_{i}} \cos \xi_{i}\right] A_{i} \\
&+ \frac{1}{v_{i}^{2}-\xi_{i}^{2}}\left[\xi_{i} e^{-v_{i}} \sin \xi_{i}-v_{i} e^{-v_{i}} \cos \xi_{i}+v_{i}\right] B_{i} .
\end{aligned}
$$


Analogously we can get the following representation for the process $\tilde{\Psi}(t)$ with $x_{\Psi}(t)=\beta \tilde{x}_{0}(t)-\alpha \tilde{x}_{0}(t-1):$

$$
\begin{aligned}
\tilde{\Psi}(t) & =\sigma^{-1}\left(x_{\Psi}(t) X_{0}(0)+b \int_{-1}^{0} x_{\Psi}(t-s-1) X_{0}(s) d s\right. \\
& \left.+\int_{0}^{t} x_{\Psi}(t-s) d W(s)\right)
\end{aligned}
$$

for $t \geq 2$; and $x_{\varphi}$ has the properties $x_{\Psi}(t)=0$ for $t \in[-1,0]$; and for $t \rightarrow \infty$ it holds

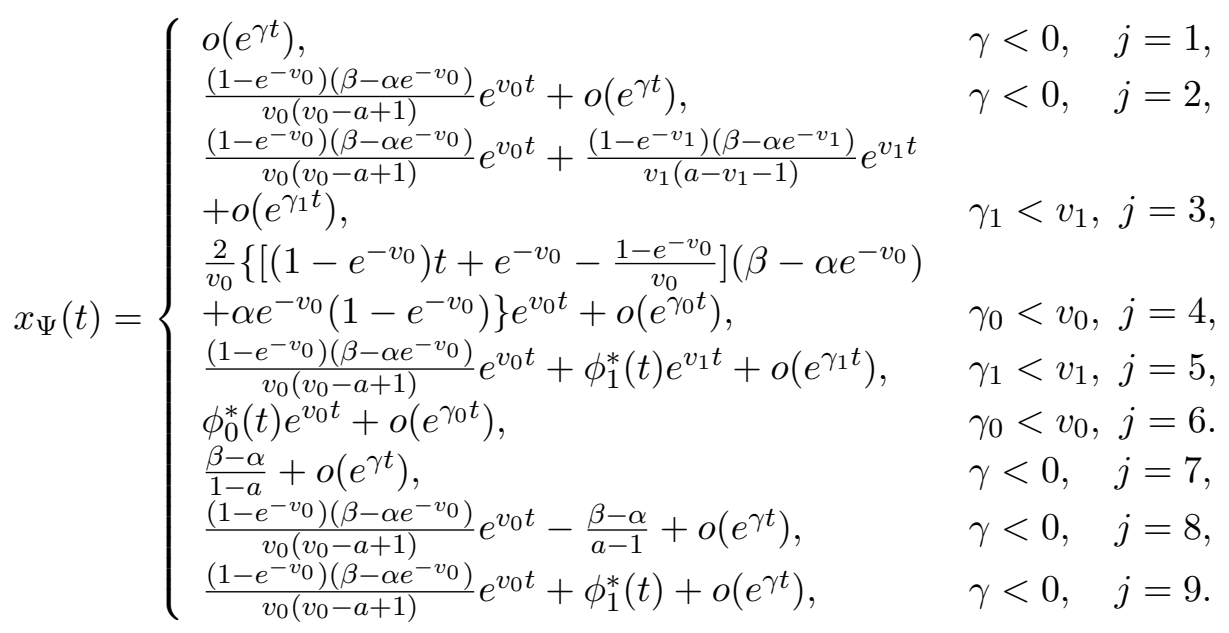

Here

$$
\begin{gathered}
\phi_{i}^{*}(t)=A_{i}^{*} \cos \xi_{i} t+B_{i}^{*} \sin \xi_{i} t, \\
A_{i}^{*}=\beta \tilde{A}_{i}-\alpha \tilde{A}_{i} e^{-v_{i}} \cos \xi_{i}-\alpha \tilde{B}_{i} e^{-v_{i}} \sin \xi_{i}, \\
B_{i}^{*}=\beta \tilde{B}_{i}-\alpha \tilde{A}_{i} e^{-v_{i}} \sin \xi_{i}-\alpha \tilde{B}_{i} e^{-v_{i}} \cos \xi_{i}, i=0,1 .
\end{gathered}
$$

The processes $\tilde{\Psi}(t)$ and $\tilde{V}(t)$ are mutually independent (by assumption, $W, V$ and $X_{0}$ are independent), and the process $\tilde{\Psi}(t)$ has a representation similar to (18). This is a consequence of the definition of $\tilde{\Psi}$ and the preceding calculations.

Then, after a series of calculations similar to those in $[2]$ and $[5,6]$ we get the following limits:

- for $(i, j) \in I_{1}$

$$
f_{i j u}= \begin{cases}\sigma^{-2}\left(\int_{0}^{\infty} x_{\Psi}^{2}(t) d t+1\right), & u=0 \\ \sigma^{-2} \int_{0}^{\infty} x_{\Psi}(t+u) x_{\Psi}(t) d t, & u \geq 2\end{cases}
$$

- for $(i, j) \in I_{2} \cup I_{3}$

$$
\begin{gathered}
\lim _{t \rightarrow \infty} e^{-v_{i} t} \tilde{\Psi}(t)=\tilde{c}_{i j} U_{i} \quad P-\text { a.s. } \\
U_{i}=X_{0}(0)+b \int_{-1}^{0} e^{-v_{i}(s+1)} X_{0}(s) d s+\int_{0}^{\infty} e^{-v_{i} s} d W(s),
\end{gathered}
$$




$$
\begin{gathered}
\tilde{c}_{0 j}=\frac{\left(1-e^{-v_{0}}\right)\left(\beta-\alpha e^{-v_{0}}\right)}{v_{0}\left(v_{0}-a+1\right)} \sigma^{-1}, \tilde{c}_{13}=\frac{\left(1-e^{-v_{1}}\right)\left(1-e^{v_{0}-v_{1}}\right)}{v_{1}\left(a-v_{1}-1\right)} \beta \sigma^{-1}, \\
\tilde{c}_{14}=\frac{2\left(1-e^{-v_{0}}\right)}{v_{0}} \beta \sigma^{-1}
\end{gathered}
$$

and as follows

$$
f_{i j u}=\frac{\tilde{c}_{i j}^{2} U_{i}^{2}}{2 v_{i}} e^{-v_{i} u}, u \geq 0 ;
$$

- for $(i, j) \in I_{4}$

$$
\begin{gathered}
\lim _{t \rightarrow \infty} t^{-1} e^{-v_{0} t} \tilde{\Psi}(t)=\tilde{c}_{0} U_{0} \quad P-\text { a.s. } \\
\tilde{c}_{0}=\frac{2\left(1-e^{-v_{0}}\right)}{v_{0}}\left(\beta-\alpha e^{-v_{0}}\right) \sigma^{-1}
\end{gathered}
$$

and

$$
f_{i j u}=\frac{\tilde{c}_{0}^{2} U_{0}^{2}}{4 v_{0}} e^{-v_{i} u}, u \geq 0
$$

- for $(i, j) \in I_{5}$

$$
\lim _{t \rightarrow \infty}\left|e^{-v_{i} t} \tilde{\Psi}(t)-U_{i j}(t)\right|=0 \quad P-\text { a.s. }
$$

where for $(i, j) \in I_{5} \backslash\{(1,6)\}$

$$
\begin{aligned}
U_{i j}(t) & =\sigma^{-1}\left(X_{0}(0) \phi_{i}^{*}(t)+b \int_{-1}^{0} \phi_{i}^{*}(t-s-1) e^{-v_{i}(s+1)} X_{0}(s) d s\right. \\
& \left.+\int_{0}^{\infty} \phi_{i}^{*}(t-s) e^{-v_{i} s} d W(s)\right), \\
U_{16}(t) & =\sigma^{-1}\left(X_{0}(0) \phi_{0}^{*}(t)+b \int_{-1}^{0} \phi_{0}^{*}(t-s-1) e^{-v_{i}(s+1)} X_{0}(s) d s\right. \\
& \left.+\int_{0}^{\infty} \phi_{0}^{*}(t-s) e^{-v_{i} s} d W(s)\right)
\end{aligned}
$$

and

$$
\begin{aligned}
f_{i j u}(T) & =\sigma^{-2} e^{v_{i} u} \int_{0}^{\infty} e^{-2 v_{i} t} U_{i}(T-t) \hat{U}_{i}(T-t) d t, u \geq 0, \\
\hat{U}_{i}(t) & =X_{0}(0) \hat{\phi}_{i}(t)+b \int_{-1}^{0} \hat{\phi}_{i}(t-s-1) e^{-v_{i}(s+1)} X_{0}(s) d s \\
& +\int_{0}^{\infty} \hat{\phi}_{i}(t-s) e^{-v_{i} s} d W(s),
\end{aligned}
$$




$$
\begin{gathered}
\hat{\phi}_{i}(t)=\hat{A}_{i} \cos \xi_{i} t+\hat{B}_{i} \sin \xi_{i} t \\
\hat{A}_{i}=A_{i}^{*} \cos \xi_{i} u-B_{i}^{*} \sin \xi_{i} u, \hat{B}_{i}=-A_{i}^{*} \sin \xi_{i} u+B_{i}^{*} \cos \xi_{i} u, i=0,1 .
\end{gathered}
$$

Here $U_{i}(t) \equiv \hat{U}_{i}(t)$ by $u=0$.

The relations (16) and (17) are proved. We continue to show the finiteness of $T(\varepsilon)$.

Because the function $x_{\Psi}(t)$ is defined similar to the function $x_{0}(t)$ (its structure and properties have been investigated, for example, in [2]), we can see that meas $\{u \in$ $\left.(2,3]: f_{i j u}=0\right\}=0$ in the cases $(i, j) \in I_{1}$ and it is obviously that $f_{i j u} \neq 0 P$-a.s. for $(i, j) \in I_{2} \cup I_{3} \cup I_{4}$.

Define for $(i, j) \in I_{5}$

$$
\bar{f}_{i j u}=\sup _{t \in(0, \infty)}\left|f_{i j u}(t)\right|, \quad \underline{f}_{i j 0}=\inf _{t \in(0, \infty)}\left|f_{i j 0}(t)\right| .
$$

It is clear that for $u=0$ and $u \geq 2$ respectively these values are positive and finite. From here and (16), (17) it follows, in particular, the finiteness of the stopping times $\tau_{\varepsilon}(n), n \geq 1$ defined by (10), because for all $(i, j) \in I_{1} \cup I_{2} \cup I_{3} \cup I_{4}$ the limits $f_{i j 0}$ are positive $P-$ a.s.

By using (16) and the definition of $\tau_{\varepsilon}(n)$ we have the next limiting equalities: - for $(i, j) \in I_{1}$

$$
\lim _{n \rightarrow \infty} \frac{\tau_{\varepsilon}(n)}{\varepsilon^{-1} c_{n}}=\lim _{\varepsilon \rightarrow 0} \frac{\tau_{\varepsilon}(n)}{\varepsilon^{-1} c_{n}}=f_{i j 0}^{-1} P_{\vartheta}-\text { a.s. }
$$

Taking into account the inequalities

$$
\int_{0}^{\tau_{\varepsilon}(n)-2-4 h} \Psi^{2}(t) d t<\varepsilon^{-1} c_{n} \leq \int_{0}^{\tau_{\varepsilon}(n)-2-3 h} \Psi^{2}(t) d t
$$

we obtain:

- for $(i, j) \in I_{2} \cup I_{3}$

$$
\begin{gathered}
e^{2 v_{i}(2+3 h)} f_{i j 0}^{-1} \leq \lim _{n \rightarrow \infty} \frac{e^{2 v_{i} \tau_{\varepsilon}(n)}}{\varepsilon^{-1} c_{n}} \leq \varlimsup_{n \rightarrow \infty} \frac{e^{2 v_{i} \tau_{\varepsilon}(n)}}{\varepsilon^{-1} c_{n}} \leq e^{4 v_{i}(1+2 h)} f_{i j 0}^{-1} P-\text { a.s. }, \\
e^{2 v_{i}(2+3 h)} f_{i j 0}^{-1} \leq \lim _{\varepsilon \rightarrow 0} \frac{e^{2 v_{i} \tau_{\varepsilon}(n)}}{\varepsilon^{-1} c_{n}} \leq \varlimsup_{\varepsilon \rightarrow 0} \frac{e^{2 v_{i} \tau_{\varepsilon}(n)}}{\varepsilon^{-1} c_{n}} \leq e^{4 v_{i}(1+2 h)} f_{i j 0}^{-1} P-\text { a.s. }
\end{gathered}
$$

and as follows

$$
\begin{gathered}
2+3 h-\frac{1}{2 v_{i}} \ln f_{i j 0}+\frac{1}{2 v_{i}} \ln \varepsilon^{-1} \leq \lim _{n \rightarrow \infty}\left[\tau_{\varepsilon}(n)-\frac{1}{2 v_{i}} \ln c_{n}\right] \leq \varlimsup_{n \rightarrow \infty}\left[\tau_{\varepsilon}(n)\right. \\
\left.-\frac{1}{2 v_{i}} \ln c_{n}\right] \leq 2(1+2 h)-\frac{1}{2 v_{i}} \ln f_{i j 0}+\frac{1}{2 v_{i}} \ln \varepsilon^{-1} P-\text { a.s. },
\end{gathered}
$$




$$
\begin{gathered}
2+3 h-\frac{1}{2 v_{i}} \ln f_{i j 0}+\frac{1}{2 v_{i}} \ln c_{n} \leq \lim _{\varepsilon \rightarrow 0}\left[\tau_{\varepsilon}(n)-\frac{1}{2 v_{i}} \ln \varepsilon^{-1}\right] \leq \varlimsup_{\varepsilon \rightarrow 0}\left[\tau_{\varepsilon}(n)\right. \\
\left.-\frac{1}{2 v_{i}} \ln \varepsilon^{-1}\right] \leq 2(1+2 h)-\frac{1}{2 v_{i}} \ln f_{i j 0}+\frac{1}{2 v_{i}} \ln c_{n} P-\text { a.s. }
\end{gathered}
$$

- for $(i, j) \in I_{4}$

$$
\begin{aligned}
e^{2 v_{i}(2+3 h)} f_{i j 0}^{-1} \leq & \lim _{n \rightarrow \infty} \frac{\tau_{\varepsilon}^{2}(n) e^{2 v_{i} \tau_{\varepsilon}(n)}}{\varepsilon^{-1} c_{n}} \leq \varlimsup_{n \rightarrow \infty} \frac{\tau_{\varepsilon}^{2}(n) e^{2 v_{i} \tau_{\varepsilon}(n)}}{\varepsilon^{-1} c_{n}} \\
& \leq e^{4 v_{i}(1+2 h)} f_{i j 0}^{-1} P-\text { a.s. } \\
e^{2 v_{i}(2+3 h)} f_{i j 0}^{-1} \leq & \lim _{\varepsilon \rightarrow 0} \frac{\tau_{\varepsilon}^{2}(n) e^{2 v_{i} \tau_{\varepsilon}(n)}}{\varepsilon^{-1} c_{n}} \leq \varlimsup_{\varepsilon \rightarrow 0} \frac{\tau_{\varepsilon}^{2}(n) e^{2 v_{i} \tau_{\varepsilon}(n)}}{\varepsilon^{-1} c_{n}} \\
& \leq e^{4 v_{i}(1+2 h)} f_{i j 0}^{-1} P-\text { a.s. }
\end{aligned}
$$

From (17) and by the definition $(10)$ of $\tau_{\varepsilon}(n)$ for all $(i, j) \in I_{5}$ we have

$$
e^{2 v_{i}(2+3 h)} \bar{f}_{i j 0}^{-1} \leq \lim _{n \rightarrow \infty} \frac{e^{2 v_{i} \tau_{\varepsilon}(n)}}{\varepsilon^{-1} c_{n}} \leq \varlimsup_{n \rightarrow \infty} \frac{e^{2 v_{i} \tau_{\varepsilon}(n)}}{\varepsilon^{-1} c_{n}} \leq e^{4 v_{i}(1+2 h)} \underline{f}_{i j 0}^{-1} P-\text { a.s. }
$$

and

$$
e^{2 v_{i}(2+3 h)} \bar{f}_{i j 0}^{-1} \leq \lim _{\varepsilon \rightarrow 0} \frac{e^{2 v_{i} \tau_{\varepsilon}(n)}}{\varepsilon^{-1} c_{n}} \leq \varlimsup_{\varepsilon \rightarrow 0} \frac{e^{2 v_{i} \tau_{\varepsilon}(n)}}{\varepsilon^{-1} c_{n}} \leq e^{4 v_{i}(1+2 h)} \underline{f}_{i j 0}^{-1} P-\text { a.s. }
$$

From (28) we obtain for every $\varepsilon>0$

$$
\begin{gathered}
2+3 h-\frac{1}{2 v_{i}} \ln \bar{f}_{i j 0}+\frac{1}{2 v_{i}} \ln \varepsilon^{-1} \leq \lim _{n \rightarrow \infty}\left[\tau_{\varepsilon}(n)-\frac{1}{2 v_{i}} \ln c_{n}\right] \leq \overline{\lim }_{n \rightarrow \infty}\left[\tau_{\varepsilon}(n)\right. \\
\left.-\frac{1}{2 v_{i}} \ln c_{n}\right] \leq 2(1+2 h)-\frac{1}{2 v_{i}} \ln \underline{f}_{i j 0}+\frac{1}{2 v_{i}} \ln \varepsilon^{-1} P-\text { a.s. }
\end{gathered}
$$

and from (29) for $n \geq 1$ if follows

$$
\begin{gathered}
2+3 h-\frac{1}{2 v_{i}} \ln \bar{f}_{i j 0}+\frac{1}{2 v_{i}} \ln c_{n} \leq \frac{\lim }{\varepsilon \rightarrow 0}\left[\tau_{\varepsilon}(n)-\frac{1}{2 v_{i}} \ln \varepsilon^{-1}\right] \leq \varlimsup_{\varepsilon \rightarrow 0}\left[\tau_{\varepsilon}(n)\right. \\
\left.-\frac{1}{2 v_{i}} \ln \varepsilon^{-1}\right] \leq 2(1+2 h)-\frac{1}{2 v_{i}} \ln \underline{f}_{i j 0}+\frac{1}{2 v_{i}} \ln c_{n} P-\text { a.s. }
\end{gathered}
$$

Note that in the cases $I_{2} \cup I_{3} \cup I_{5}$ we have

$$
\lim _{n \rightarrow \infty} \frac{\tau_{\varepsilon}(n)}{\ln c_{n}}=\lim _{\varepsilon \rightarrow 0} \frac{\tau_{\varepsilon}(n)}{\ln \varepsilon^{-1}}=\frac{1}{2 v_{i}} P-\text { a.s. }
$$


Put $\delta_{\varepsilon}(n)=\tau_{\varepsilon}(n) k_{n} h$.

Now we are able to show the finiteness of the stopping time $\nu_{\varepsilon}$. From (16), (21), (22) and (26) with $P$-probability one we have the relations:

- for $(i, j) \in I_{1}$

$$
\lim _{n \rightarrow \infty} \frac{1}{c_{n}} \int_{2}^{\delta_{\varepsilon}(n)} \Psi(t-2-3 h) \Psi(t) d t=\left(\varepsilon f_{i j 0}\right)^{-1} f_{i j(2+3 h)} ;
$$

- for $(i, j) \in I_{2} \cup I_{3} \cup I_{4}$

$$
\begin{gathered}
e^{4 v_{i}}\left(\varepsilon f_{i j 0}\right)^{-1}\left|f_{i j(2+3 h)}\right| \leq \lim _{n \rightarrow \infty}\left|\frac{1}{c_{n}} \int_{2}^{\delta_{\varepsilon}(n)} \Psi(t-2-3 h) \Psi(t) d t\right| \\
\leq \lim _{n \rightarrow \infty}\left|\frac{1}{c_{n}} \int_{2}^{\delta_{\varepsilon}(n)} \Psi(t-2-3 h) \Psi(t) d t\right| \leq e^{2 v_{i}(2+3 h)}\left(\varepsilon f_{i j 0}\right)^{-1}\left|f_{i j(2+3 h)}\right| .
\end{gathered}
$$

Consider the cases $(i, j) \in I_{5}$. For all $u \geq 2$ and $(i, j) \in I_{5}$ the functions $f_{i j u}(T)$ of $T$ are periodic with corresponding periods $\Delta>1$ and each of them has at most two roots on every interval of the lengths $\Delta$. Denote these roots for $u=2+3 h$ as $t_{m}(i, j), m \leq 2$ on the set $(0, \Delta]$. Then define $V_{i j}$ to be the union of open disjoint neighborhoods with the radius less then $1 / 6$ for all roots $t_{m}(i, j)+N \Delta, m=1,2, N \geq 0$ and put

$$
\mathcal{R}_{i j}^{+}=(0, \infty) \backslash V_{i j}
$$

Define

$$
\begin{gathered}
f_{i j u}^{*}=\inf _{t \in \mathcal{R}_{i j}^{+}}\left|f_{i j u}(t)\right|, \\
Q_{n}(i, j)=\left\{k=\overline{1,3}: n h-k h \in \mathcal{R}_{i j}^{+}\right\}, \\
r_{i j}(n)=\arg \max _{k \in Q_{n}(i j)}\left|f_{i j(2+3 h)}(n h-k h)\right| .
\end{gathered}
$$

By the continuity of $f_{i j u}(\cdot)$ we have $f_{i j u}^{*}>0$ for $u=0$ and $u \geq 2$. Note that for any $h \in(0,1 / 3]$ and $(i, j) \in I_{5}$ the sets $Q_{n}(i, j)$ are non-empty and for $n$ large enough from (17) we have

$$
r_{i j}(n)=\arg \max _{k \in Q_{n}(i, j)}\left|e^{-2 v_{i}(n h-k h)} \int_{0}^{n h-k h} \Psi(t-2-3 h) \Psi(t) d t\right| \quad P \text { - a.s., }
$$

besides by the definition of $Q_{n}(i, j)$ for $n$ large enough with $P$-probability one

$$
f_{i j(2+3 h)}^{*} \leq\left|f_{i j(2+3 h)}\left(n h-r_{i j}(n) h\right)\right| \leq \bar{f}_{i j(2+3 h)}
$$

and

$$
f_{i j(2+3 h)}^{*} \leq\left|e^{-2 v_{i}\left(n h-r_{i j}(n) h\right)} \int_{0}^{n h-r_{i j}(n) h} \Psi(t-2-3 h) \Psi(t) d t\right| \leq \bar{f}_{i j(2+3 h)} .
$$


Then for $(i, j) \in I_{5}$ with $P$-probability one we obtain the following relations

$$
\begin{gathered}
\varlimsup_{n \rightarrow \infty}\left|e^{-2 v_{i} n h} \int_{0}^{n h-r_{n} h} \Psi(t-2-3 h) \Psi(t) d t\right| \\
=\varlimsup_{n \rightarrow \infty} e^{-2 v_{i} r_{n} h}\left|e^{-2 v_{i}\left(n h-r_{n} h\right)} \int_{0}^{n h-r_{n} h} \Psi(t-2-3 h) \Psi(t) d t\right| \leq e^{-2 v_{i} h} \bar{f}_{i j(2+3 h)}, \\
\lim _{n \rightarrow \infty}\left|e^{-2 v_{i} n h} \int_{0}^{n h-r_{n} h} \Psi(t-2-3 h) \Psi(t) d t\right| \geq \lim _{n \rightarrow \infty} e^{-2 v_{i} r_{i j}(n) h} \\
\cdot\left|e^{-2 v_{i}\left(n h-r_{i j}(n) h\right)} \int_{0}^{n h-r_{i j}(n) h} \Psi(t-2-3 h) \Psi(t) d t\right| \geq e^{-6 v_{i} h} f_{i j(2+3 h)}^{*}
\end{gathered}
$$

and as follows for all $\varepsilon>0$

$$
\begin{aligned}
& e^{-6 v_{i} h} f_{i j(2+3 h)}^{*} \leq \lim _{n \rightarrow \infty}\left|e^{-2 v_{i} \tau_{\varepsilon}(n)} \int_{0}^{\tau_{\varepsilon}(n)-k_{n} h} \Psi(t-2-3 h) \Psi(t) d t\right| \\
\leq & \varlimsup_{n \rightarrow \infty}\left|e^{-2 v_{i} \tau_{\varepsilon}(n)} \int_{0}^{\tau_{\varepsilon}(n)-k_{n} h} \Psi(t-2-3 h) \Psi(t) d t\right| \leq e^{-2 v_{i} h} \bar{f}_{i j(2+3 h)} .
\end{aligned}
$$

In such a way for the cases $(i, j) \in I_{5}$ from (28) and (35) with $P$-probability one we have

$$
\begin{gathered}
e^{4 v_{i}}\left(\varepsilon \bar{f}_{i j 0}\right)^{-1} f_{i j(2+3 h)}^{*} \leq \lim _{n \rightarrow \infty} \frac{1}{c_{n}}\left|\int_{2}^{\delta_{\varepsilon}(n)} \tilde{\Psi}(t-2-3 h) \tilde{\Psi}(t) d t\right| \\
\leq \lim _{n \rightarrow \infty} \frac{1}{c_{n}}\left|\int_{2}^{\delta_{\varepsilon}(n)} \tilde{\Psi}(t-2-3 h) \tilde{\Psi}(t) d t\right| \leq e^{2 v_{i}(2+3 h)}\left(\varepsilon \underline{f}_{i j 0}\right)^{-1} \bar{f}_{i j(2+3 h) .}
\end{gathered}
$$

The finiteness of $\nu_{\varepsilon}$ follows from the definition $\nu_{\varepsilon},(33),(34),(36)$ and the condition (14) on the sequences $\left(c_{n}\right)$ and $\left(\kappa_{n}\right)$.

Thus the finiteness of the stopping times $T(\varepsilon)$ is established.

Let us estimate the mean square deviation of $\theta_{\varepsilon}^{*}$. From (11) and by definitions of the stopping time $\nu_{\varepsilon}$ and $\rho$ it follows that for all $\vartheta \in \mathcal{R}^{2}$

$$
\begin{gathered}
E_{\vartheta}\left(\theta_{\varepsilon}^{*}-\theta\right)^{2}=E_{\vartheta} G_{\varepsilon}^{-2}\left(\nu_{\varepsilon}\right) \zeta_{\varepsilon}^{2}\left(\nu_{\varepsilon}\right) \leq \frac{\varepsilon^{2}}{\rho} E_{\vartheta} \frac{1}{\kappa_{\nu_{\varepsilon}}^{2}} \zeta_{\varepsilon}^{2}\left(\nu_{\varepsilon}\right) \\
\leq \frac{\varepsilon^{2}}{\rho} \sum_{n \geq 1} \frac{1}{\kappa_{n}^{2}} E_{\vartheta} \zeta_{\varepsilon}^{2}(n) \leq \frac{9\left(2+\bar{s}^{2}\right) \varepsilon}{\rho} \sum_{n \geq 1} \frac{c_{n}}{\kappa_{n}^{2}}=\varepsilon .
\end{gathered}
$$

Thus the first property $\mathrm{I} .1^{o}$ of the sequential plans $\left(T(\varepsilon), \theta_{\varepsilon}^{*}\right)$ in Theorem 3.1 is proved. 
In order to establish the second property note that similar to $(33),(34),(36)$ for all $n \geq 1$ we can prove P-a.s.

- for $(i, j) \in I_{1}$

$$
\lim _{\varepsilon \rightarrow 0} \varepsilon \int_{0}^{\delta_{\varepsilon}(n)} \Psi(t-2-3 h) \Psi(t) d t=f_{i j 0}^{-1} f_{i j(2+3 h)} c_{n} ;
$$

- for $(i, j) \in I_{2} \cup I_{3} \cup I_{4}$

$$
\begin{aligned}
& e^{4 v_{i}} f_{i j 0}^{-1}\left|f_{i j(2+3 h)}\right| c_{n} \leq \lim _{\varepsilon \rightarrow 0} \varepsilon\left|\int_{0}^{\delta_{\varepsilon}(n)} \Psi(t-2-3 h) \Psi(t) d t\right| \\
& \leq \varlimsup_{\varepsilon \rightarrow 0} \varepsilon\left|\int_{0}^{\delta_{\varepsilon}(n)} \Psi(t-2-3 h) \Psi(t) d t\right| \leq e^{2 v_{i}(2+3 h)} f_{i j 0}^{-1}\left|f_{i j(2+3 h)}\right| c_{n} ;
\end{aligned}
$$

- for $(i, j) \in I_{5}$

$$
\begin{aligned}
& e^{4 v_{i}} \bar{f}_{i j 0}^{-1} f_{i j(2+3 h)}^{*} c_{n} \leq \frac{\lim _{\varepsilon \rightarrow 0} \varepsilon}{\delta^{\prime}} \varepsilon \int_{0}^{\delta_{\varepsilon}(n)} \Psi(t-2-3 h) \Psi(t) d t \mid \\
& \leq \varlimsup_{\varepsilon \rightarrow 0} \varepsilon\left|\int_{0}^{\delta_{\varepsilon}(n)} \Psi(t-2-3 h) \Psi(t) d t\right| \leq e^{2 v_{i}(2+3 h)} \underline{f}_{i j 0}^{-1} \bar{f}_{i j(2+3 h)} c_{n} .
\end{aligned}
$$

Analogously to [11] from the definition of $\nu_{\varepsilon}$ and from (37)-(39) we can see that for $\varepsilon$ small enough and $(i, j) \in I_{1} \cup I_{2} \cup I_{3} \cup I_{4} \cup I_{5}$

$$
\nu_{i j}^{\prime} \leq \nu_{\varepsilon} \leq \nu_{i j}^{\prime \prime} \quad P_{\vartheta}-\text { a.s. }
$$

where

$$
\begin{gathered}
\nu_{i j}^{\prime}=\max \left\{\inf \left\{n \geq 1: c_{n} / \kappa_{n}>g_{i j}^{\prime}\right\}-1,1\right\}, \\
\nu_{i j}^{\prime \prime}=\inf \left\{n \geq 1: c_{n} / \kappa_{n}>g_{i j}^{\prime \prime}\right\}, \\
g_{i j}^{\prime}= \begin{cases}\rho^{1 / 2} f_{i j 0}\left|f_{i j(2+3 h)}^{-1}\right|, & (i, j) \in I_{1}, \\
\rho^{1 / 2} e^{-2 v_{i}(2+3 h)} f_{i j 0} \mid f_{i j(2+3 h)}^{-1}, & (i, j) \in I_{2} \cup I_{3} \cup I_{4}, \\
\rho^{1 / 2} e^{-2 v_{i}(2+3 h)} \underline{f}_{i j 0} \bar{f}_{i j(2+3 h)}^{-1}, & (i, j) \in I_{5},\end{cases} \\
g_{i j}^{\prime \prime}= \begin{cases}g_{i j}^{\prime}, & (i, j) \in I_{1}, \\
\rho^{1 / 2} e^{-4 v_{i}} f_{i j 0}\left|f_{i j(2+3 h)}^{-1}\right|, & (i, j) \in I_{2} \cup I_{3} \cup I_{4}, \\
\rho^{1 / 2} e^{-4 v_{i}} \bar{f}_{i j 0}\left(f_{i j(2+3 h)}^{*}\right)^{-1}, & (i, j) \in I_{5} .\end{cases}
\end{gathered}
$$

Now from (12), (21), (25), (27), (31) and (40) the second I. $2^{o}$ assertion of Theorem 3.1 follows: 
- for $(i, j) \in I_{1}$ by

$$
f_{i j 0}^{-1} c_{\nu_{i j}^{\prime}} \leq \varliminf_{\varepsilon \rightarrow 0} \varepsilon T(\varepsilon) \leq \varlimsup_{\varepsilon \rightarrow 0} \varepsilon T(\varepsilon) \leq f_{i j 0}^{-1} c_{\nu_{i j}^{\prime \prime}} P-\text { a.s.; }
$$

- for $(i, j) \in I_{2} \cup I_{3}$ by

$$
\begin{gathered}
2+3 h-\frac{1}{2 v_{i}} \ln f_{i j 0}+\frac{1}{2 v_{i}} \ln c_{\nu_{i j}^{\prime}} \leq \frac{\lim }{\varepsilon \rightarrow 0}\left[T(\varepsilon)-\frac{1}{2 v_{i}} \ln \varepsilon^{-1}\right] \\
\leq \varlimsup_{\varepsilon \rightarrow 0}\left[T(\varepsilon)-\frac{1}{2 v_{i}} \ln \varepsilon^{-1}\right] \leq 2(1+2 h)-\frac{1}{2 v_{i}} \ln f_{i j 0}+\frac{1}{2 v_{i}} \ln c_{\nu_{i j}^{\prime \prime}} P-\text { a.s. }
\end{gathered}
$$

- for $(i, j) \in I_{4}$ by

$$
\begin{gathered}
e^{2 v_{0}(2+3 h)} f_{i j 0}^{-1} c_{\nu_{i j}^{\prime}} \leq \varliminf_{\varepsilon \rightarrow 0} \varepsilon T^{2}(\varepsilon) e^{2 v_{0} T(\varepsilon)} \leq \varlimsup_{\varepsilon \rightarrow 0} \varepsilon T^{2}(\varepsilon) e^{2 v_{0} T(\varepsilon)} \\
\leq e^{4 v_{0}(1+2 h)} f_{i j 0}^{-1} c_{\nu_{i j}^{\prime \prime}} P-\text { a.s.; }
\end{gathered}
$$

- for $(i, j) \in I_{5}$ by

$$
\begin{gathered}
2+3 h-\frac{1}{2 v_{i}} \ln \bar{f}_{i j 0}+\frac{1}{2 v_{i}} \ln c_{\nu_{i j}^{\prime}} \leq \frac{\lim }{\varepsilon \rightarrow 0}\left[T(\varepsilon)-\frac{1}{2 v_{i}} \ln \varepsilon^{-1}\right] \\
\leq \varlimsup_{\varepsilon \rightarrow 0}\left[T(\varepsilon)-\frac{1}{2 v_{i}} \ln \varepsilon^{-1}\right] \leq 2(1+2 h)-\frac{1}{2 v_{i}} \ln \underline{f}_{i j 0}+\frac{1}{2 v_{i}} \ln c_{\nu_{i j}^{\prime \prime}} P-\text { a.s. }
\end{gathered}
$$

Thus the proof of part I of Theorem 3.1 is finished.

In order to prove the second assertion II of Theorem 3.1 note that according to (33), (34) and (36)

$$
\varlimsup_{n \rightarrow \infty} c_{n}\left|G_{\varepsilon}^{-1}(n)\right|<\infty P-\text { a.s. }
$$

and from (13), (14) it follows that

$$
\sum_{n \geq 1} \frac{1}{c_{n}}<\infty
$$

In view of the form for the deviation of the estimators $\theta_{\varepsilon}(n)$ from $\vartheta$ it suffices to establish the next limiting equality

$$
\lim _{n \rightarrow \infty} \frac{1}{c_{n}} \zeta_{\varepsilon}(n)=0 P-\text { a.s. }
$$

which follows from (11), as well as Chebychev's inequality and by the Borel-Cantelli lemma.

Therefore strong consistency of the estimators $\theta_{\varepsilon}(n), \varepsilon>0$ is obtained. 


\subsection{Proof of Theorem 3.2}

Firstly we show the finiteness of the stopping times $\tilde{T}(\varepsilon)$.

We start by calculating for $u=0$ and $u \geq 1$ the limits

$$
\lim _{T \rightarrow \infty} \frac{1}{T} \int_{1}^{T} \tilde{\Delta} Y(t) \tilde{\Delta} Y(t-u) d t=f(u) P-\text { a.s. }
$$

in the stationary case and

$$
\lim _{T \rightarrow \infty}\left|\frac{1}{e^{2 v_{0} T}} \int_{1}^{T} \tilde{\Delta} Y(t) \tilde{\Delta} Y(t-u) d t-f_{u}(T)\right|=0 P-\text { a.s. }
$$

in the periodic case, where $f(u)$ is random function of $u$ and $f_{u}(T)$ are periodic functions of $T$ for all $u \geq 0$.

From (2) we have

$$
\tilde{\Delta} Y(t)=\tilde{X}(t)+\tilde{\Delta} V(t), t \geq 1
$$

By assumption the processes $\tilde{X}(t)$ and $\tilde{\Delta} Y(t)$ are mutually independent. Similar to the proof of Theorem 3.1 we can get the following limiting relations using the definition of the process $\tilde{X}(t)$ :

- in the stationary case

$$
f(u)= \begin{cases}\int_{0}^{\infty} \tilde{x}_{0}^{2} d t+1, & u=0 \\ \int_{0}^{\infty} \tilde{x}_{0}(t+u) \tilde{x}_{0}(t) d t, & u \geq 1\end{cases}
$$

- in the periodic case

$$
\begin{aligned}
& f_{u}(T)=e^{-v_{0} u} \int_{0}^{\infty} e^{-2 v_{0} t} U_{0}^{*}(T-t) U_{u}^{*}(T-t) d t, u \geq 0, \\
& U_{u}^{*}(t)=X_{0}(0) \tilde{\phi}_{u}^{*}(t)+b \int_{-1}^{0} \tilde{\phi}_{u}^{*}(t-s-1) e^{-v_{0}(s+1)} X_{0}(s) d s \\
& +\int_{0}^{\infty} \tilde{\phi}_{u}^{*}(t-s) e^{-v_{0} s} d W(s) \\
& \tilde{\phi}_{u}^{*}(t)=\tilde{A}_{u}^{*} \cos \xi_{0} t+\tilde{B}_{u}^{*} \sin \xi_{0} t \\
& \tilde{A}_{u}^{*}=\tilde{A}_{0} \cos \xi_{0} u-\tilde{B}_{0} \sin \xi_{0} u, \tilde{B}_{u}^{*}=\tilde{B}_{0} \cos \xi_{0} u-\tilde{A}_{0} \sin \xi_{0} u .
\end{aligned}
$$

By the definition of $\xi_{0}$ we can see that functions $f_{u}(T)$ are periodic with the period $\Delta>1$. Note that $f(0)>0$ and $0<\underline{f}_{0}=\inf _{T} f_{0}(T)<\sup _{T} f_{0}(T)=\bar{f}_{0}<\infty$.

The relations (41), (42) and therefore the finiteness of the times $\tilde{\tau}_{\varepsilon}(n), n \geq 1, \varepsilon>$ 0 are established.

From $(41),(42)$ and by the definition of the stopping times $\tilde{\tau}_{\varepsilon}(n)$ we have the next limiting relations: 
- in the stationary case

$$
\lim _{n \rightarrow \infty} \frac{\tilde{\tau}_{\varepsilon}(n)}{\varepsilon^{-1} c_{n}}=\lim _{\varepsilon \rightarrow 0} \frac{\tilde{\tau}_{\varepsilon}(n)}{\varepsilon^{-1} c_{n}}=(2 f(0))^{-1} P-\text { a.s. } ;
$$

- in the periodic case for any $\varepsilon>0$

$$
\begin{gathered}
e^{2 v_{0}(2+5 \tilde{h})}\left[\varepsilon\left(1+e^{2 v_{0} \tilde{h}}\right) \bar{f}_{0}\right]^{-1} \leq \lim _{n \rightarrow \infty} c_{n}^{-1} e^{2 v_{0} \tilde{\tau}_{\varepsilon}(n)} \\
\leq \varlimsup_{n \rightarrow \infty} c_{n}^{-1} e^{2 v_{0} \tilde{\tau}_{\varepsilon}(n)} \leq e^{4 v_{0}(1+3 \tilde{h})}\left[\varepsilon\left(1+e^{2 v_{0} \tilde{h}}\right) \underline{f}_{0}\right]^{-1} P-\text { a.s. }
\end{gathered}
$$

and for $n \geq 1$

$$
\begin{gathered}
e^{4 v_{0}(1+3 \tilde{h})}\left[\left(1+e^{2 v_{0} \tilde{h}}\right) \bar{f}_{0}\right]^{-1} c_{n} \leq \underline{\lim }_{\varepsilon \rightarrow 0} \varepsilon e^{2 v_{0} \tilde{\tau}_{\varepsilon}(n)} \leq \varlimsup_{\varepsilon \rightarrow 0} \varepsilon e^{2 v_{0} \tilde{\tau}_{\varepsilon}(n)} \\
\leq e^{2 v_{0}(2+7 \tilde{h})}\left[\left(1+e^{2 v_{0} \tilde{h}}\right) \underline{f}_{0}\right]^{-1} c_{n} P-\text { a.s. }
\end{gathered}
$$

From (43), (44) in the periodic case for $\varepsilon>0$

$$
\begin{gathered}
2(1+3 \tilde{h})-\frac{1}{2 v_{0}} \ln \left(1+e^{2 v_{0} \tilde{h}}\right)-\frac{1}{2 v_{0}} \ln \bar{f}_{0}+\frac{1}{2 v_{0}} \ln \varepsilon^{-1} \\
\leq \lim _{n \rightarrow \infty}\left[\tilde{\tau}_{\varepsilon}(n)-\frac{1}{2 v_{0}} \ln c_{n}\right] \leq \varlimsup_{n \rightarrow \infty}\left[\tilde{\tau}_{\varepsilon}(n)-\frac{1}{2 v_{0}} \ln c_{n}\right] \leq 2+7 \tilde{h} \\
-\frac{1}{2 v_{0}} \ln \left(1+e^{2 v_{0} \tilde{h}}\right)-\frac{1}{2 v_{0}} \ln \underline{f}_{0}+\frac{1}{2 v_{0}} \ln \varepsilon^{-1} P-\text { a.s. }
\end{gathered}
$$

and for $n \geq 1$

$$
\begin{gathered}
2(1+3 \tilde{h})-\frac{1}{2 v_{0}} \ln \left(1+e^{2 v_{0} \tilde{h}}\right)-\frac{1}{2 v_{0}} \ln \bar{f}_{0}+\frac{1}{2 v_{0}} \ln c_{n} \\
\leq \lim _{\varepsilon \rightarrow 0}\left[\tilde{\tau}_{\varepsilon}(n)-\frac{1}{2 v_{0}} \ln \varepsilon^{-1}\right] \leq \varlimsup_{\varepsilon \rightarrow 0}\left[\tilde{\tau}_{\varepsilon}(n)-\frac{1}{2 v_{0}} \ln \varepsilon^{-1}\right] \\
\leq 2+7 \tilde{h}-\frac{1}{2 v_{0}} \ln \left(1+e^{2 v_{0} \tilde{h}}\right)-\frac{1}{2 v_{0}} \ln \underline{f}_{0}+\frac{1}{2 v_{0}} \ln c_{n} P-\text { a.s. }
\end{gathered}
$$

From (41), (43) we can obtain in the stationary case

$$
\begin{gathered}
\lim _{n \rightarrow \infty} \frac{\varepsilon}{c_{n}} \int_{1}^{\tilde{\tau}_{\varepsilon}(n)} \tilde{\Delta} Y(t-u) \tilde{\Delta} Y(t) d t=\lim _{\varepsilon \rightarrow 0} \frac{\varepsilon}{c_{n}} \int_{1}^{\tilde{\tau}_{\varepsilon}(n)} \tilde{\Delta} Y(t-u) \tilde{\Delta} Y(t) d t \\
=(2 f(0))^{-1} f(u), u \geq 1 \quad P-\text { a.s. }
\end{gathered}
$$


and

$$
\begin{aligned}
& \lim _{n \rightarrow \infty} \frac{\varepsilon}{c_{n}} \tilde{G}_{\varepsilon}(n)=\lim _{\varepsilon \rightarrow 0} \frac{\varepsilon}{c_{n}} \tilde{G}_{\varepsilon}(n)=\tilde{G}(\tilde{h}) P-\text { a.s. } \\
& \tilde{G}(\tilde{h})=(2 f(0))^{-1}\left(\begin{array}{ll}
f(2+5 \tilde{h}) & f(1+\tilde{h}) \\
f(2+6 \tilde{h}) & f(1+6 \tilde{h})
\end{array}\right) .
\end{aligned}
$$

Similar to the Case I we can see that meas $\left\{u \in\left[\tilde{h}_{0}, 1 / 5\right]: f(u)=0\right\}=0$ and meas $\left\{u \in\left[\tilde{h}_{0}, 1 / 5\right]: \operatorname{det} \tilde{G}(u)=0\right\}=0$. As follows $\operatorname{det} \tilde{G}(\tilde{h}) \neq 0 P-$ a.s. From here, (14), (15) and (48) we have the finiteness of the times $\tilde{\nu}_{\varepsilon}$ in the stationary case.

Put

$$
\tilde{G}(T, \tilde{h})=\left(\begin{array}{ll}
f_{2+5 \tilde{h}}(T) & e^{-2 v_{0}} f_{1+5 \tilde{h}}(T) \\
f_{2+6 \tilde{h}}(T) & e^{-2 v_{0}} f_{1+6 \tilde{h}}(T)
\end{array}\right)
$$

From (42) in the periodic case it follows that the matrices $\tilde{G}(T, s)$ are the limits of the matrix functions $\tilde{f}(T, s)=\frac{1}{e^{2 v_{0} T}} G^{*}(T, s)$ in the almost surely sense:

$$
\lim _{T \rightarrow \infty}|\tilde{f}(T, s)-\tilde{G}(T, s)|=0, s \geq 0 P-\text { a.s. }
$$

The matrix functions $\tilde{G}(T, s)$ are periodic with the period $\Delta>1$ and according to the definition of functions $f_{u}(T), u \geq 0$ the equation

$$
\operatorname{det} \tilde{G}(T, s)=0
$$

has at most four roots $\tilde{t}_{m}, m=\overline{1,4}$ on the set $(0, \Delta]$ for any $s$. Put $\tilde{\delta}_{\varepsilon}(n)=$ $\tilde{\tau}_{\varepsilon}(n)-\tilde{k}_{n} \tilde{h}$. Note that in the periodic case by the definition of $\tilde{G}_{\varepsilon}(n)(15)$ and from (42) analogously to the proof of Theorem 3.1 we can get the following relations

$$
\begin{gathered}
\lim _{n \rightarrow \infty}\left|\frac{1}{e^{2 v_{0} \tilde{\delta}_{\varepsilon}(n)}} \tilde{G}_{\varepsilon}(n)-\tilde{G}\left(\tilde{\delta}_{\varepsilon}(n), \tilde{h}\right)\right| \\
=\lim _{\varepsilon \rightarrow 0}\left|\frac{1}{e^{2 v_{0} \tilde{\delta}_{\varepsilon}(n)}} \tilde{G}_{\varepsilon}(n)-\tilde{G}\left(\tilde{\delta}_{\varepsilon}(n), \tilde{h}\right)\right|=0 P-\text { a.s. }
\end{gathered}
$$

and for some constants $\tilde{g}_{1}, \tilde{g}_{2}$

$$
\begin{gathered}
0<\tilde{g}_{1}=\lim _{n \rightarrow \infty}\left\|\tilde{G}^{-1}\left(\tilde{\delta}_{\varepsilon}(n), \tilde{h}\right)\right\| \leq \varlimsup_{n \rightarrow \infty}\left\|\tilde{G}^{-1}\left(\tilde{\delta}_{\varepsilon}(n), \tilde{h}\right)\right\|=\tilde{g}_{2}<\infty, \\
0<\tilde{g}_{1}=\varliminf_{\varepsilon \rightarrow 0}\left\|\tilde{G}^{-1}\left(\tilde{\delta}_{\varepsilon}(n), \tilde{h}\right)\right\| \leq \varlimsup_{\varepsilon \rightarrow 0}\left\|\tilde{G}^{-1}\left(\tilde{\delta}_{\varepsilon}(n), \tilde{h}\right)\right\|=\tilde{g}_{2}<\infty .
\end{gathered}
$$

From $(14),(15),(44),(50)$ and (51) the finiteness of times $\tilde{\nu}_{\varepsilon}$ in the periodic case follows.

Thus the finiteness of the stopping times $\tilde{T}(\varepsilon)$ is established. 
The property I. $1^{o}$ of the sequential estimators $(\tilde{T}(\varepsilon), \tilde{\vartheta}(\varepsilon))$ and the strong consistency of the estimators $\tilde{\vartheta}_{\varepsilon}(n)$ may be proved similar to the proof of Theorem 3.1.

Now we find the limiting low and upper bounds for the duration time $\tilde{T}(\varepsilon)$ of our sequential estimation. Put for $k=1,2$

$$
\begin{gathered}
\tilde{\nu}(k)=\inf \left\{n \geq 1: \quad c_{n} / \kappa_{n}>\tilde{g}(k)\right\}-1, \\
\nu^{*}(k)=\inf \left\{n \geq 1: c_{n} / \kappa_{n}>g^{*}(k)\right\}, \\
\tilde{g}(1)=g^{*}(1)=2 f(0) \tilde{\rho}^{1 / 2}\left\|\tilde{G}^{-1}(\tilde{h})\right\|, \\
\tilde{g}(2)=\tilde{\rho}^{1 / 2} \tilde{g}_{1} e^{-4 v_{0}(1+3 \tilde{h})}\left(1+e^{2 v_{0} \tilde{h}}\right) \underline{f}_{0}, \\
g^{*}(2)=\tilde{\rho}^{1 / 2} \tilde{g}_{2} e^{-2 v_{0}(2+\tilde{h})}\left(1+e^{2 v_{0} \tilde{h}}\right) \bar{f}_{0} .
\end{gathered}
$$

By the definition of $\tilde{\nu}_{\varepsilon}$ and from (43), (45), (48), (50), (52) it follows that for $\varepsilon$ small enough

- in the stationary case

$$
\tilde{\nu}(1) \leq \tilde{\nu}_{\varepsilon} \leq \nu^{*}(1)
$$

- in the periodic case

$$
\tilde{\nu}(2) \leq \tilde{\nu}_{\varepsilon} \leq \nu^{*}(2) .
$$

From (15), (43), (47), (53) and (54) the assertion I. $2^{\circ}$ of Theorem 3.2 follows: -in the stationary case

$$
(2 f(0))^{-1} c_{\tilde{\nu}(1)} \leq \lim _{\varepsilon \rightarrow 0} \varepsilon \tilde{T}(\varepsilon) \leq \varlimsup_{\varepsilon \rightarrow 0} \varepsilon \tilde{T}(\varepsilon) \leq(2 f(0))^{-1} c_{\nu^{*}(1)} P-\text { a.s. } ;
$$

- in the periodic case

$$
\begin{aligned}
& 2(1+3 \tilde{h})-\frac{1}{2 v_{0}} \ln \left(1+e^{2 v_{0} \tilde{h}}\right)-\frac{1}{2 v_{0}} \ln \bar{f}_{0}+\frac{1}{2 v_{0}} \ln c_{\tilde{\nu}(2)} \\
\leq & \lim _{\varepsilon \rightarrow 0}\left[\tilde{T}(\varepsilon)-\frac{1}{2 v_{0}} \ln \varepsilon^{-1}\right] \leq \varlimsup_{\varepsilon \rightarrow 0}\left[\tilde{T}(\varepsilon)-\frac{1}{2 v_{0}} \ln \varepsilon^{-1}\right] \leq 2+7 \tilde{h} \\
& -\frac{1}{2 v_{0}} \ln \left(1+e^{2 v_{0} \tilde{h}}\right)-\frac{1}{2 v_{0}} \ln \underline{f}_{0}+\frac{1}{2 v_{0}} \ln c_{\nu^{*}(2)} P-\text { a.s. }
\end{aligned}
$$

Remark 4 It should be pointed out that one could obtain the following limiting equalities for $(i, j) \in I_{1}$ in Problem $I$

$$
\lim _{\varepsilon \rightarrow 0} \varepsilon \tilde{T}(\varepsilon)=f_{i j 0}^{-1} c_{\nu_{i j}^{*}} P-\text { a.s. }
$$

and in stationary case in Problem $I I$

$$
\lim _{\varepsilon \rightarrow 0} \varepsilon \tilde{T}(\varepsilon)=(2 f(0))^{-1} c_{\nu^{*}(1)} P-\text { a.s. }
$$

if the magnitudes $\rho^{1 / 2} \varepsilon^{-1} c_{n}\left|G_{\varepsilon}^{-1}(n)\right|$ and $\tilde{\rho}^{1 / 2} \varepsilon^{-1} c_{n}\left|\tilde{G}_{\varepsilon}^{-1}(n)\right|$ in the definitions of $\nu_{\varepsilon}$ and $\tilde{\nu}_{\varepsilon}$ respectively were replaced by the nearest integer from above and the sequences $\left(c_{n}\right)$ and $\left(\kappa_{n}\right)$ were chosen in such a way that the relation $c_{n} / \kappa_{n}$ were fractional for all $n \geq 1$. 


\section{References}

[1] M. Arato, Linear Stochastic Systems with Constant Coefficients. A Statistical Approach (Berlin, Heidelberg, New York, Springer Verlag, 1982)

[2] A.A. Gushchin and U. Küchler, Asymptotic inference for a linear stochastic differential equation with time delay, Bernoulli 5(6) (1999) 1059-1098.

[3] V. Kolmanovskii and A. Myshkis, Applied Theory of Functional Differential Equations (Kluwer Acad. Pabl., 1992)

[4] U. Küchler and M. Soerensen, Exponential families of stochastic processes (New York, Heidelberg, Springer Verlag, 1997)

[5] U. Küchler and V. Vasil'iev, On sequential parameter estimation for some linear stochastic differential equations with time delay, Sequential Analysis 20 (3) (2001) 117-146.

[6] U. Küchler and V. Vasiliev, Sequential identification of linear dynamic systems with memory, Statistical Inference for Stochastic Processes 8 (1) (2005) 1-24.

[7] R.Sh. Liptser and A.N. Shiryaev, Statistics of Random Processes (New York, Heidelberg, Springer-Verlag, 1977)

[8] R.Sh. Liptser and A.N. Shiryaev, Theory of martingales (Moscow, Nauka, 1986)

[9] X. Mao, Stochastic Differential Equations and Application (Harwood Publishing Chichester, 1997)

[10] S.E-A. Mohammed, Stochastic Differential Systems with Memory: Theory, Examples and Applications, Probability and Statistics (Birkhäuser, 1996) 42 1-77.

[11] V.A. Vasiliev and V.V. Konev, On sequential identification of linear dynamic systems in continuous time by noisy observations. Probl. of Contr. and Inform. Theory 16 (2) (1987) 101-112. 